\title{
Ultrasensitive gene fusion detection reveals fusion variant associated tumor heterogeneity
}

\section{Baifeng Zhang}

University of Hong Kong https://orcid.org/0000-0003-1966-2285

\section{Zhengbo Song}

Zhejiang Cancer Hospital

Chloe Yufan Bao

Karolinska Institutet

\section{Chunwei Xu}

Department of Pathology, Fujian Cancer Hospital, Fujian Medical University

\section{Wenxian Wang}

Department of Chemotherapy, Zhejiang Cancer Hospital

Hoi Yee Chu

Karolinska Institutet

Chenyu Lu

City University of Hong Kong

Hongxiang Wang

Shanghai Changzheng Hospital

\section{Siyu Bao}

Karolinska Institute https://orcid.org/0000-0002-6703-5393

\section{Zhenyu Gong}

Shanghai Changzheng Hospital

\section{Hoi Yee Keung}

Karolinska Institutet

\section{Maggie Chow}

Karolinska Institutet

\section{Yiping Zhang}

Karolinska Institutet

\section{Wah Cheuk}

Queen Elizabeth Hospital

\section{Mengsu Yang}

City University of Hong Kong

\section{William Cho}

Queen Elizabeth Hospital

Juxiang Chen 
Shanghai Changzheng Hospital

\section{Zongli Zheng ( $\square$ zongli.zheng@ki.se)}

Karolinska Institutet https://orcid.org/0000-0003-4849-4903

Article

Keywords:

Posted Date: August 1st, 2020

DOI: https://doi.org/10.21203/rs.3.rs-39138/v1

License: (c) (1) This work is licensed under a Creative Commons Attribution 4.0 International License. Read Full License 


\section{Ultrasensitive gene fusion detection reveals fusion variant associated tumor heterogeneity}

2

3 Baifeng Zhang ${ }^{1,10}$, Zhengbo Song ${ }^{2,3,10}$, Chloe $\mathrm{Bao}^{1,4,10}$, Chunwei $\mathrm{Xu}^{5,10}$, Wenxian Wang ${ }^{2,3}$, Athena Hoi

4 Yee $\mathrm{Chu}^{1}$, Chenyu $\mathrm{Lu}^{4,8}$, Hongxian Wang ${ }^{6}$, Siyu Bao ${ }^{1,4}$, Zhenyu Gong ${ }^{6}$, Hoi Yee Keung ${ }^{1}$, Maggie Zi-

5 Ying Chow $^{1}$, Yiping Zhang ${ }^{2,3}$, Wah Cheuk ${ }^{7}$, Mengsu Yang ${ }^{4,8}$, William Chi Shing Choo ${ }^{9 *}$, Juxiang

6 Chen $^{6^{*}}$, Zongli Zheng ${ }^{1,4,8^{*}}$

7

8 1. Ming Wai Lau Centre for Reparative Medicine, Karolinska Institutet, Hong Kong, China.

9 2. Department of Medical Oncology, Cancer Hospital of the University of Chinese Academy of 10 Sciences (Zhejiang Cancer Hospital), Hangzhou 310022, China.

11 3. Institute of Cancer and Basic Medicine, Chinese Academy of Sciences, Hangzhou 310022, China.

12 4. Department of Biomedical Sciences, City University of Hong Kong, Hong Kong, China.

13 5. Department of Pathology, Fujian Cancer Hospital, Fuzhou, 350014, China.

14 6. Department of Neurosurgery, Shanghai Changzheng Hospital, Second Military Medical 15 University, Shanghai 200003, China.

16 7. Department of Pathology, Queen Elizabeth Hospital, Hong Kong, China.

17 8. Biotechnology and Health Centre, City University of Hong Kong Shenzhen Research Institute, 18 Shenzhen, China.

19 9. Department of Clinical Oncology, Queen Elizabeth Hospital, Hong Kong, China.

20 10. These authors contributed equally.

22 *Correspondence should be addressed to zhengzongli@gmail.com, juxiangchen@126.com, or

23 williamcscho@gmail.com 
Abstract (words 150)

Gene fusions are common drivers and therapeutic targets in cancers, but clinical-grade bioinformatics

27 callers are lacking. Here we introduce a novel method SplitFusion, which is fast by leveraging BWA-

28 MEM split alignments, can detect cryptic splice site fusions, and can infer frame-ness and exon-

29 boundary alignments for functional prediction and minimizing false-positives. SplitFusion

30 demonstrates superior sensitivity, specificity, accuracy and consumes minimal computing resources.

31 In our study of 1,076 formalin-fixed paraffin-embedded lung cancer samples, SplitFusion detected not

32 only common fusions ( $A L K 4.7 \%$, ROS1 $2.0 \%$ and RET $1.1 \%$ ) with various partners, but also rare

33 (KLC1-ALK, CD74-NRG1, and TPR-NTRK1) and novel (FGFR3-JAKMP1, CLIP2-BRAF, and

34 ITPR2-ETV6) fusions. In 35 glioblastoma samples, SplitFusion-Target detected six (17\%) EGFR vIII

35 (exons 2-7 deletion) cases. Furthermore, we find that the $E M L 4-A L K$ variant 3 is significantly

36 associated with occurrence of multiple breakpoint-defined subclones, namely high intratumor

37 heterogeneity. In conclusion, SplitFusion is well-suited for clinical use and for studying fusion-

38 defined tumor heterogeneity. 
40 Chromosomal rearrangements or gene fusions that lead to novel or overexpressed oncoproteins have

41 been identified across different human cancers ${ }^{1}$. The cure for leukemia harboring $B C R-A B L^{2}$ and $42 P M L-R A R A^{3}$ gene fusions represented the early success in the era of precision medicine. Beyond 43 blood cancers, discoveries in gene fusions have continued to revolutionize the management of solid tumors, including $\mathrm{ALK}^{4}$ and $\mathrm{ROS}^{5}$ in lung cancer, TMPRSS2 in about $50 \%$ of prostate cancer ${ }^{6}$, FGFRs in diverse cancers ${ }^{7}$, and NTRK fusions with FDA approved drugs regardless of tissue type ${ }^{8}$.

Clinical diagnosis of gene fusions has been challenging in both wet lab and bioinformatics. The prevalence of gene fusions in The Cancer Genome Atlas study was estimated about $16 \%$ overall $^{9}$ and $3 \%$ for those involving promising therapeutic targets (ranging from 0 to $12.9 \%$ across different tissue types $)^{10}$. These RNA-seq analyses are powerful in gene fusion discoveries. However, the associated low specificities are problematic for clinical diagnosis because non-trivial efforts are needed to filter away clinically insignificant gene fusions (e.g. $37 \%$ of the identified 25,664 fusions could not be validated in genomic data ${ }^{9}$ ). Furthermore, alternative RNA splicing and exon deletion caused by alterations at the genomic level, which may be therapeutically important such as $E G F R v I I I^{11}$, are not detectable by most fusion detection software. Numerous software have been developed to detect gene fusions for next-generation sequencing (NGS) data, such as EricScript ${ }^{12}$, Lumpy ${ }^{13}$ and STAR-

57 Fusion $^{14}$, the latter being the best-performing one among 23 different software ${ }^{14}$. Most of these software are designed for analyzing high-quality RNA-seq data ${ }^{14}$, while none of them, to our knowledge, is able to infer fusion frame-ness and to judge exon-boundary alignment (for filtering functionally insignificant or clinically irrelevant fusions); to report targeted alternative splicing and exon deletion events; and also well-suited for clinical diagnosis where formalin-fixed paraffinembedded (FFPE) samples are most accessible but often highly degraded. We have previously designed a NGS target enrichment method, termed Anchored Multiplex PCR (AMP), for scalable and efficient detection of gene fusions, without prior knowledge of the fusion partners ${ }^{15}$. For broad clinical and research applications, a high-performing bioinformatics caller is still 
67 required. Here, we introduce a novel algorithm named SplitFusion (https://github.com/Zheng-NGS-

68 Lab/SplitFusion), a clinical-grade fusion detection algorithm with high research potentials.

69 SplitFusion is fast by leveraging the chimeric alignment (split-read) of BWA-MEM ${ }^{17}$ for RNA

70 sequencing data, and demonstrates higher sensitivity and specificity than current well-known

71 software. Furthermore, SplitFusion features highly desirable abilities in clinical reporting, including

72 the capabilities to infer fusion transcript frame-ness and exon-boundary alignments; to calculate

73 number of unique DNA fragment ligation sites; and to customize target filtering and output. In 1,076

74 lung cancer and 35 glioblastoma FFPE samples, SplitFusion not only detected common, rare but also

75 novel fusions, as well as intragenic exon deletions. Lastly, SplitFusion reveals that breakpoint hot-

76 zone in EML4-ALK variant 3 (v3) is associated with a high intratumor heterogeneity, as defined by

77 fusion breakpoint subclones. An intriguing question arises from recent clinical observations is that

78 patients with lung tumors positive for the v3 of EML4-ALK fusion is associated with poorer clinical

79 outcomes than lung cancer patients with other EML4-ALK variants ${ }^{18-20}$. Using SplitFusion, we found

80 that $E M L 4-A L K \mathrm{v} 3$ was significantly associated with a high tumor heterogeneity in terms of

81 breakpoint-defined subclones - likely an underappreciated phenomenon that warrants further

82 investigations.

83

84 Results

85 SplitFusion algorithm design

86 SplitFusion can use both regular RNA-seq and anchored multiplex targeted enrichment like the

87 Anchored Multiplex PCR (Fig. 1a) NGS data. SplitFusion consists of the following major

88 computation steps. 
a. Anchored multiplex target enrichment
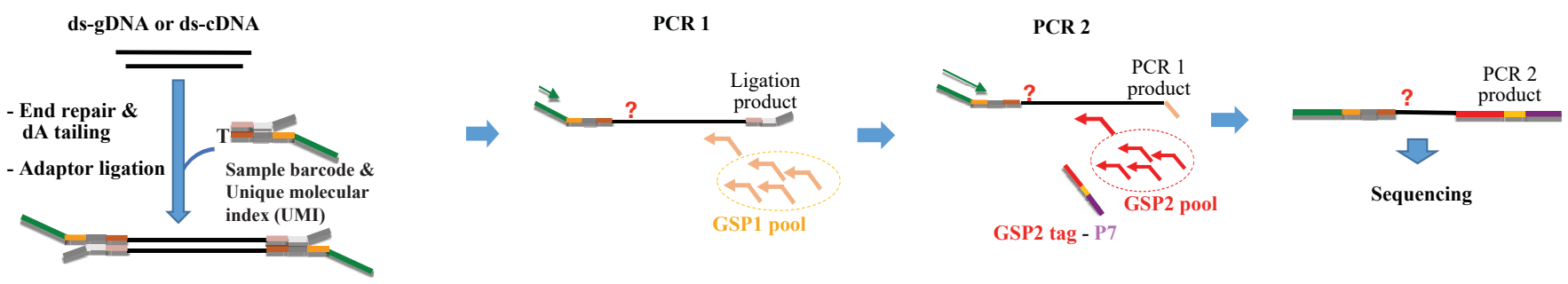

b. Reference alignment \& de-duplication

Molecular barcoded reads

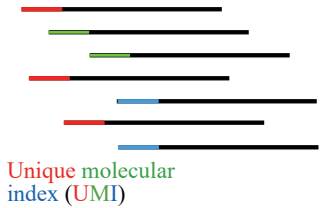

Raw FASTQ

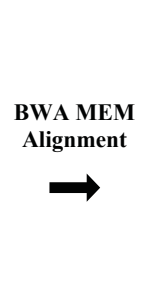

A MEM

$\rightarrow$

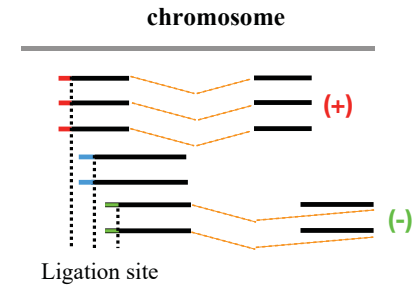

Raw BAM
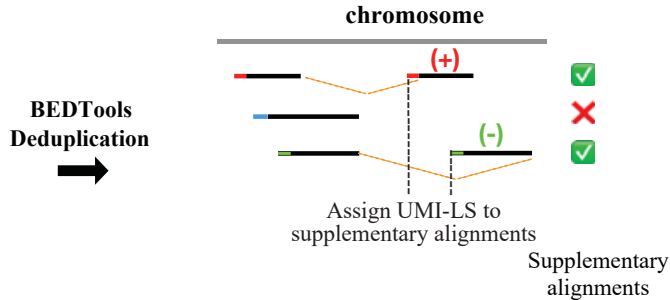

Consolidated BAM

c. CIGAR transformation \& candidate breakpoint calling

Supplementary alignments

QNAME
LEN

\begin{tabular}{|llllllc|}
\hline ReadID/2 & 86 & chr2 & 29446361 & 29446396 & + & 36M50S \\
ReadID/2 & 86 & chr2 & 42492057 & 42492091 & - & 17H35M34H \\
\hline
\end{tabular}

S: soft clip; M: mapped; H: hard clip

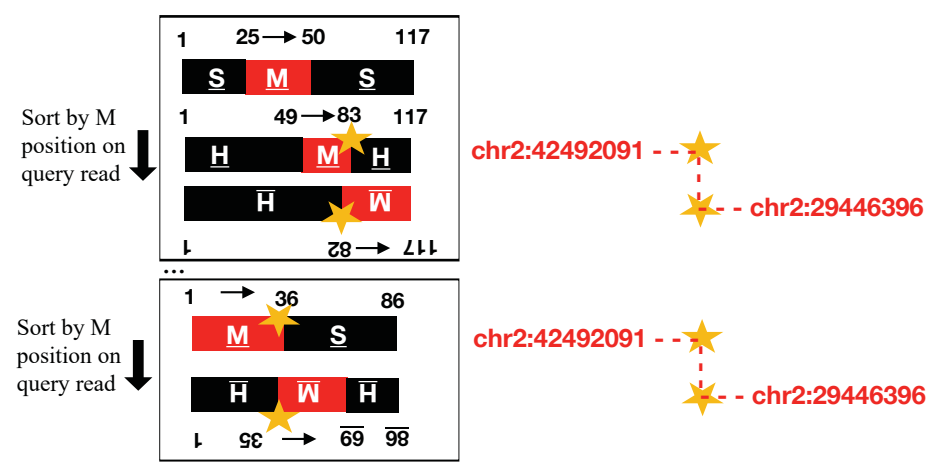

e. Breakpoint gene annotation

f. In-frame calculation

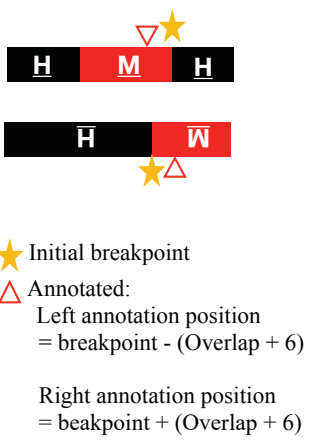

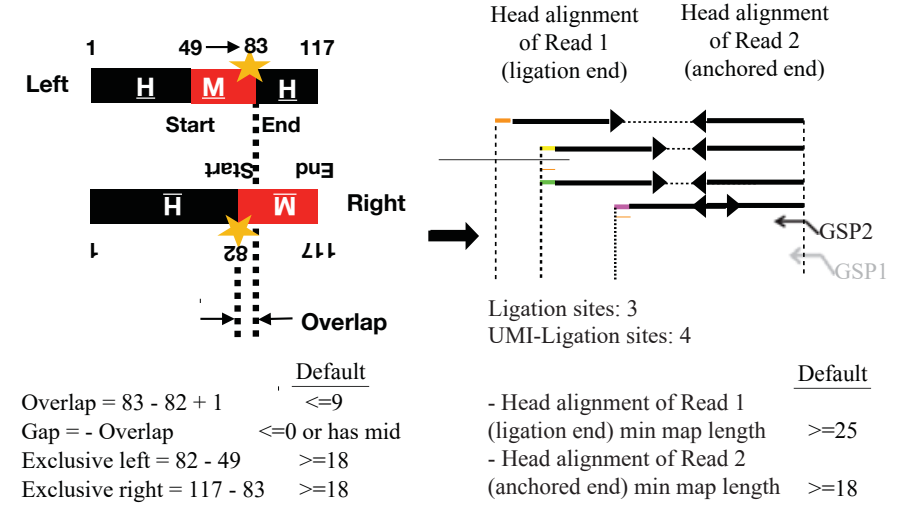

h. Result reporting \& visualization
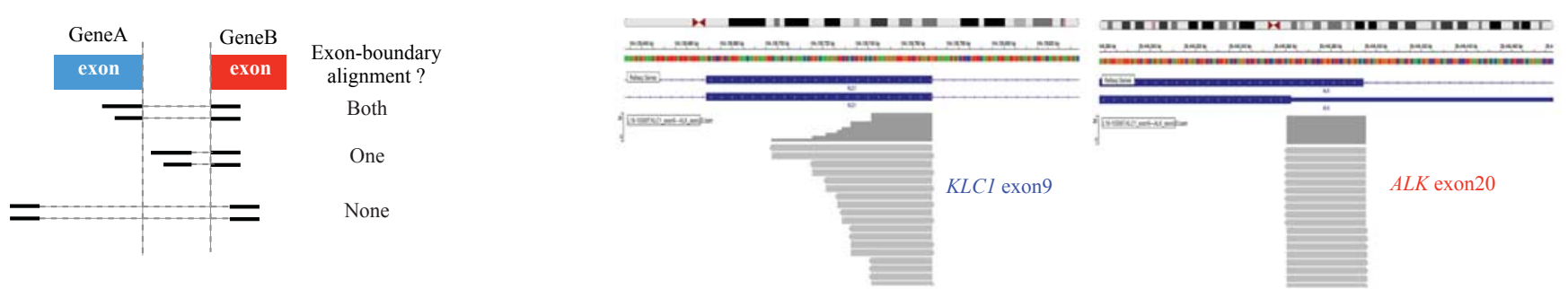

Figure 1. The framework of SplitFusion algorithm design. (a) Next-generation sequencing (NGS) library construction using the anchored multiplex PCR. Double-stranded cDNA and gDNA were subjected to end-repair, dA-tailing, and ligation of a half-functional Y adaptor, which contains sample barcode and unique molecular identifier (UMI). Two rounds of hemi-nested multiplex PCR reactions were used to enrich any sequences (denoted in red question marks) downstream of the gene specific primers (GSPs), resulting in NGS library for sequencing. (b) NGS FASTQ data was de-multiplexed by sample barcodes, followed by adaptor trimming. Different colors represent different UMIs tagged to different molecules. The data was aligned to reference using BWA-MEM, and de-duplicated based on a combination of unique UMI and ligation site (UMI-LS). Supplementary alignments were assigned with the same UMI-LS as their Read 1 starting alignments. Reads with supplementary alignments (SA) were extracted. (c) The CIGARs of reads with supplementary alignments were transformed. For the same read ID, all alignments were sorted by their mapping positions (M) on the query read, and the candidate breakpoint(s) was inferred. (d) The initial candidate breakpoints were filtered by maximum overlapping length, maximum gap, minimum exclusive mapping lengths of partner alignments, and the minimum mapping lengths of ligation end and anchored end alignments; and further subjected to gene annotation (e), frame-ness calculation (f), exon-boundary alignment judgement (g), and reporting and visualization $(\mathbf{h})$. 
Reference alignment and deduplication.

SplitFusion leverages the fast and widely used genome mapping software BWA-MEM ${ }^{17}$, which generates supplementary alignments (the SA tag in SAM specification: other canonical alignments in a chimeric alignment) for a query sequencing read containing two or more parts mapped to different locations of the reference, i.e. multiple alignments (Fig. 1b). When genome (instead of transcriptome) is used as the reference for RNA-seq reads, these different parts can be different genes, neighboring exons or cryptic splice site containing sequences. SplitFusion recognizes FASTQ or BAM file in the specified directory as input. If the BAM file (BWA MEM aligned) exists, the Kickstart mode will be initiated, bypassing the FASTQ to BAM reference alignment step. Then, deduplication / consolidation of the BAM is performed based on a combination of a unique molecular identifier (UMI) and the aligned chromosomal position of the ligation site of a DNA fragment (LS). One unique UMI-LS read (with all its split alignments) will be kept among the duplicated UMI-LS reads, resulting in a consolidated BAM file (Fig. 1b).

\section{CIGAR transformation}

SplitFusion parses the CIGAR strings, orientates and sorts chimeric parts (split-read) by their segment order in the query read (Fig. 1c). To decipher the alignment construct of a query read, CIGAR is first parsed by soft clipped (S, unmapped but not trimmed), mapped (M), and hard clipped (H, unmapped and trimmed) segments, and their lengths. Short insertion and deletions (1D/1I) in CIGAR are removed and the mapped segment (M) length is adjusted accordingly. SplitFusion then uses these $\mathrm{S} / \mathrm{M} / \mathrm{H}$ lengths of all split alignments from the same read, taking their respective alignment orientations (plus or minus strand) into account, and query read lengths as available from BEDTools ${ }^{21}$ to calculate query read start and end positions of the different split alignments. SplitFusion uses the mapping chromosomal position of ligation starting site for deduplication (based on UMI-LS) and also for final reporting on the number of partner ends. However, for cDNA fragments, the heading sequences for adaptor ligation are often partial exonic sequences (followed by coding sequences of their upstream/downstream exons) and are too short to be mapped to the reference genome. 
117 ligation site mapping position (encoded in read ID) for later accurate calculation of the number of

118 partner ends (ligation sites). SplitFusion then sorts all the split alignments from the same query read

119 by their original segment orders on the query read.

120

121 Candidate breakpoint calling.

122 From the transformed split-read alignments, SplitFusion infers breakpoint candidates. For any two

123 neighboring alignments on the same query read, if they align to different chromosomes or the same

124 chromosome but distant at $>750,000$ bases (the largest intron size used by BLAT ${ }^{22}$ ), they are deemed

125 candidate fusion partners and output to separate files (left and right). The partners are then merged by

126 their read ID, and their junction-corresponding chromosomal alignment positions are deemed as

127 candidate breakpoints (Fig. 1c). For a typical two-split alignment read, the merged new record has the

128 construct "left query start - left query end [breakpoint 1] --- right query start [breakpoint 2] - right

129 query end". For a read consists of three or more alignments (as long as the length of sequencing read

130 can accommodate), such as those involving intra-gene neighboring exons of fusion partner genes, the

131 leftmost and rightmost alignments are merged and the new record has the construct "leftmost query

132 start - nearest end to junction [breakpoint 1] --- nearest start to junction [breakpoint 2] - rightmost

133 query end". The middle alignments between the leftmost and rightmost are output to a separate file

134 (mid) for downstream analyses.

135

Initial breakpoint filtering

137 As an initial step to minimize false positive results, SplitFusion filters the candidate breakpoints by

138 minimum alignment length, minimum non-overlapping length of split parts, maximum gap length,

139 and minimal number of ligation site (UMI-LS) (Fig. 1d). SplitFusion can apply different minimum

140 mapping lengths on different partner alignments. Typically for anchored end NGS data, this feature

141 allows for a high fusion calling specificity by specifying a high mapping length value (default 25; Fig.

142 1d) on the ligation end, and also for a high fusion calling sensitivity by specifying a short mapping

143 length (default 18) on the anchored end (additional specificity on the anchored end was already 
144 imposed in the wet lab protocol by using outer gene-specific primers [GSP1s; Fig. 1a] which were

145 however not present in NGS data).

146

147 Breakpoint gene annotation, frame-ness, exon boundary, further filtering and target reporting

148 Next, SplitFusion annotates candidate breakpoints with gene names, exon numbers, and cDNA

149 positions (Fig. 1e). It is common that split alignments of the same query read share a few identical

150 ending DNA bases, which are "double counted" typically because they belong to Gene A exon and is

151 also identical to partner Gene B intron (adjacent to the fusion exon of Gene B), largely due to the

152 canonical splice junction sequences ${ }^{23}$. These "double counted" overlapping alignment segments (as a

153 result of BWA alignment seed-extension searching) need to be accounted for in order to accurately

154 calculate fusion frame-ness. SplitFusion tackles this issue by temporally shifting the breakpoint

155 positions by 6 bases (default) inwards to the center of the alignments, for both of the involved split

156 alignments, in order to annotate the breakpoints with correct cDNA positions and exon number by

157 annotation software such as ANNOVAR ${ }^{24}$ or $\operatorname{SnpEff}^{25}$. The middle alignments, if exist, are also

158 annotated. If target gene panel BED file is specified in the initial configuration, at the beginning of

159 this step, the 'Target' mode will be employed and only those alignments involving the targeted genes,

160 together with any potential partners, will be annotated. Following annotation, SplitFusion infers the

161 frame-ness of fusion transcript (in-frame/out-frame or not applicable; Fig. 1f) and judges whether the

162 breakpoints are on known exon boundary (both/one/none) according to RefSeq ${ }^{26}$ (Fig. 1g).

163 Optionally, a backend "whitelist" database can be used for targeted output to report alternative

164 splicing (e.g. for MET exon 14 skipping), exon deletion (e.g. EGFR $v I I I$ exons 2-7 deletion) and gene

165 truncation (e.g. FGFR1/2/3 exon 18 truncation), as well as a "blacklist" database to remove recurring

166 false-positive calls.

167

Result reporting and visualization

169 SplitFusion outputs a final fusion summary, including exact breakpoints, number of unique supporting

170 reads (according to UMI), number of partner ends (i.e. ligation starting site, LS), frame status, exon-

171 boundary status, fusion gene names (with transcript ID) and exon numbers. Lastly, if a fusion is 
detected, ten fusion-supporting split-reads (in FASTQ) will be randomly extracted from the

173 consolidated BAM, saved as a text file that can be examined manually, and also converted into BAM

174 file that can be visualized with a third party software or with an IGV API (Fig. 1h).

\section{Benchmarking performance versus other software}

177 To evaluate the performance of SplitFusion, we tested 46 cancer cases with known clinical diagnoses.

178 FFPE tissue sections from 10 ALK positive lung cancers, 1 EWSRl fusion positive sarcoma, and 35

$179 A L K$ fusion negative lung cancer cases (as confirmed by routine fluorescence in situ hybridization

180 [FISH] assay. Extended Data Fig. 1) were subjected to a 19-gene lung panel (Extended Data Tab.

1) targeted RNA-seq assay using $\mathrm{AMP}^{15}$. We compared four fusion detection software, namely

EricScript ${ }^{12}$, Lumpy ${ }^{13}$, STAR-Fusion ${ }^{14}$ and the present SplitFusion. We down-sampled 11 fusionpositive sample datasets to different sizes of $3 \mathrm{M}, 1 \mathrm{M}, 500 \mathrm{~K}, 100 \mathrm{~K}, 50 \mathrm{~K}, 10 \mathrm{~K}, 7.5 \mathrm{~K}$, and $5 \mathrm{~K}$ reads randomly, with 21 replicates of each size, generating a total of 1,848 datasets that were analyzed by the four software (Fig. 2a). With 500K or more reads, all software showed a sensitivity of $100 \%$ except EricScript $($ mean $=45 \%)$, but varied levels of unconfirmed or clinically insignificant fusion calls (EricScript and Lumpy: >100 per dataset as they do not include filtering steps by default; STARFusion: 5 - 14; SplitFusion: 2 - 3) (Fig. 2a). By purposely reducing the size of datasets gradually, the sensitivities of the software started to differentiate (at 10K-read: EricScript 11\%, Lumpy 57\%, STARFusion 74\%, SplitFusion 85\%; at 5K-read: EricScript 9\%, Lumpy 37\%, STAR-Fusion 61\%, SplitFusion 78\%); and unconfirmed or non-significant fusions were at the minimal level for STAR-

192 Fusion and SplitFusion (Fig. 2b). For the overall accuracy based on the previous varying read-cutoff precision-recall area under curve (AUC) ${ }^{14}$, SplitFusion showed the highest AUCs among the software across all different dataset sizes (Fig. 2c). Indeed, in the smallest 5K-read datasets, mean AUCs for EricScript, Lumpy, STAR-Fusion and SplitFusion were 0.01, 0.18, 0.48, and 0.63, respectively. As for computing resources, STAR-Fusion requires $\sim 30$ GB RAM, which was much higher than those of Lumpy ( 10 GB) and EricScript and SplitFusion (both at $\sim 4$ GB - a typical computation cluster configuration: 4 GB RAM per thread) (Fig. 2d). For the largest 3M-read datasets, the computation times were $0.65 \mathrm{~h}, 0.51 \mathrm{~h}, 0.46 \mathrm{~h}$ and $0.42 \mathrm{~h}$ for EricScript, Lumpy, STAR-Fusion and SplitFusion, 
a
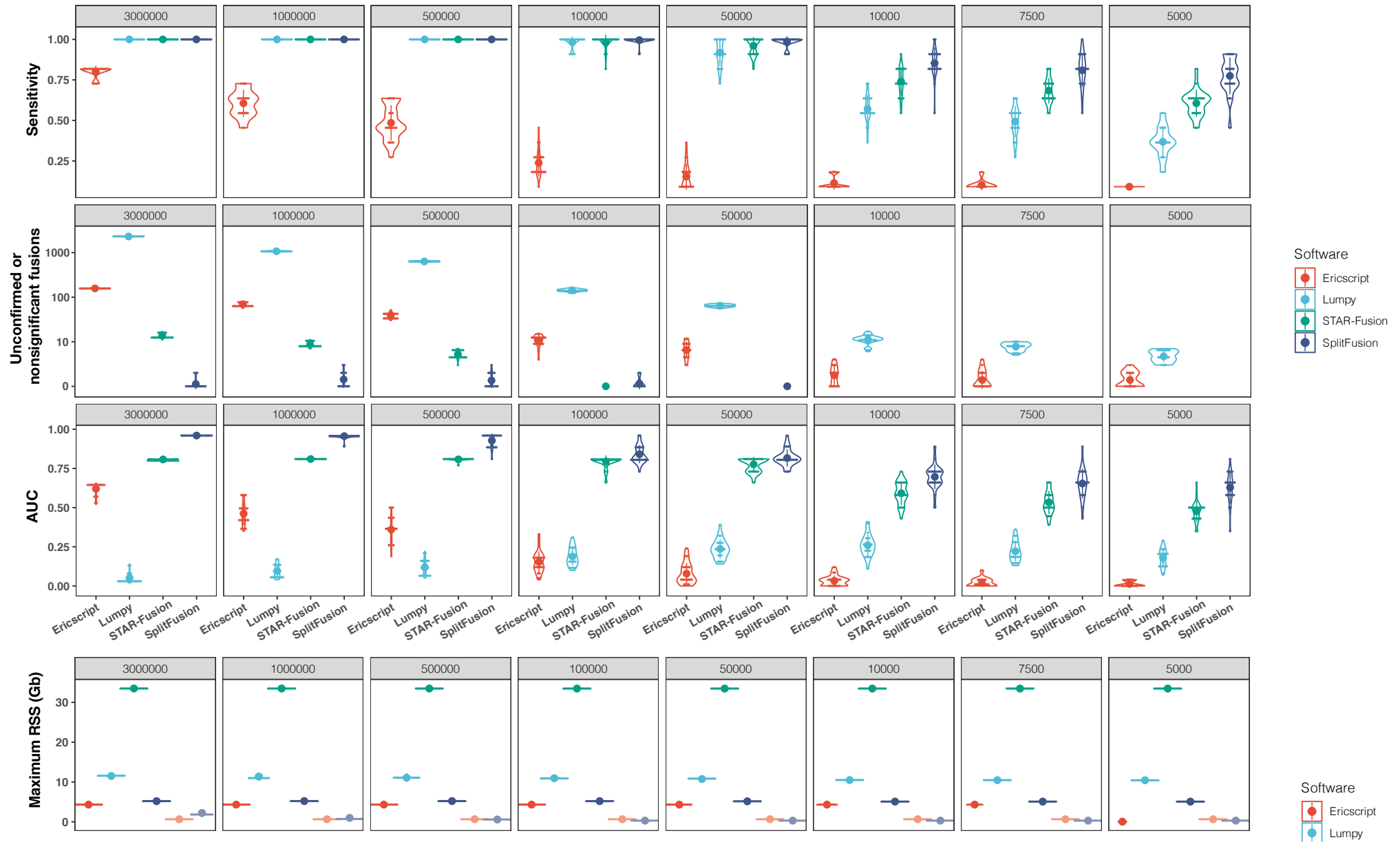

e

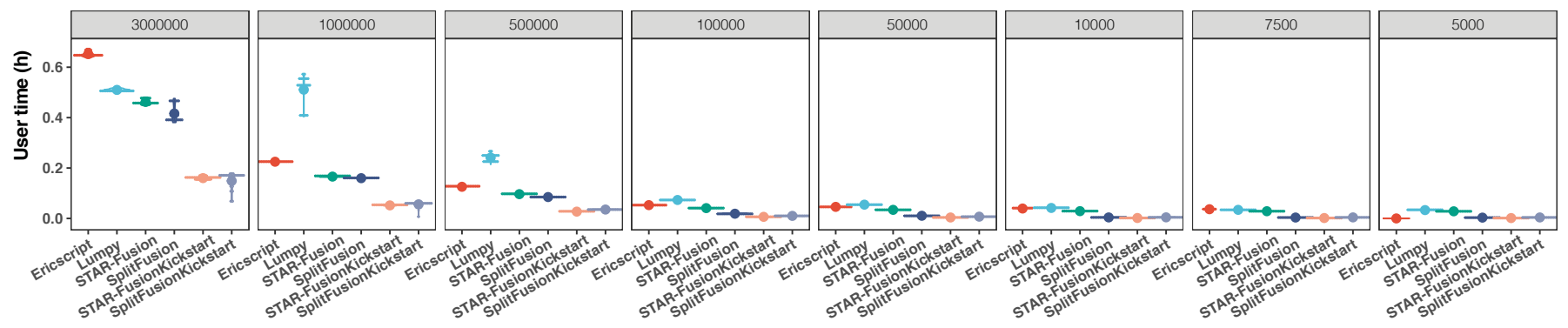

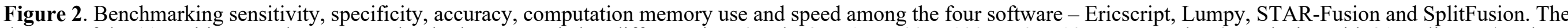

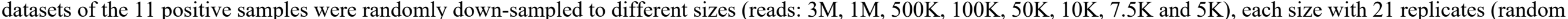

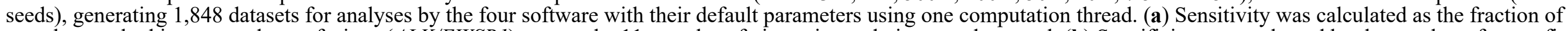

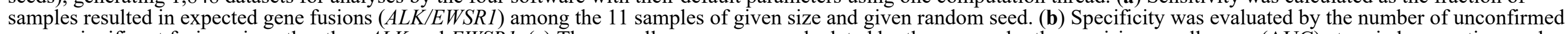

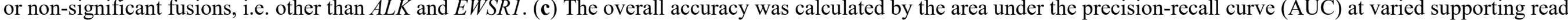

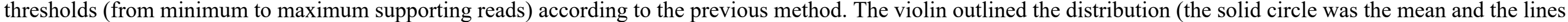

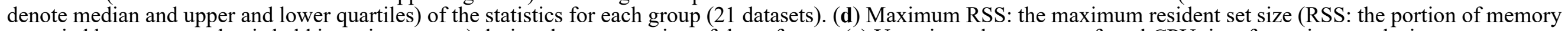
occupied by a process that is held in main memory) during the computation of the software. (e) User time: the amount of total CPU time for a given analysis. 
respectively (Fig. 2e). It is worth noting that both STAR-Fusion and SplitFusion have Kickstart

201 modes that can skip reference mapping and start from BAM, which reduced user time by $>50 \%$ (Fig. $2022 \mathbf{e})$.

203

204 Among the 35 ALK fusion negative lung cancer samples, EricScript and Lumpy reported (by default 205 without filtering) 39 and 46 unconfirmed or clinically insignificant fusions, respectively; whereas

206 STAR-Fusion reported five such fusions involving tumor suppressor gene $C D K N 2 A$, and SplitFusion 207 reported two NCOA4-RET cases with the NCOA4 breakpoint aligned on exon boundary (Fig. 3 and 208 Extended Data Fig. 2). With the Target mode, SplitFusion can report targeted outputs, such as 209 specific alternative splicing, exon-skipping and deletions. Moreover, SplitFusion can be tailored to 210 filter recurrent false positives. To demonstrate this feature, we specified EGFR vIII (exons 2 - 7 211 deletion) - a known oncogenesis driver in glioblastoma - in the target output file when analyzing

212 FFPE samples from 35 glioblastoma cases (mean age $54.8 \mathrm{y}$, male 65.7\%). SplitFusion-Target 213 reported six EGFR $v I I I$ cases (Fig. 3 \& Extended Data Fig. 3). 
Lung cancer cohort

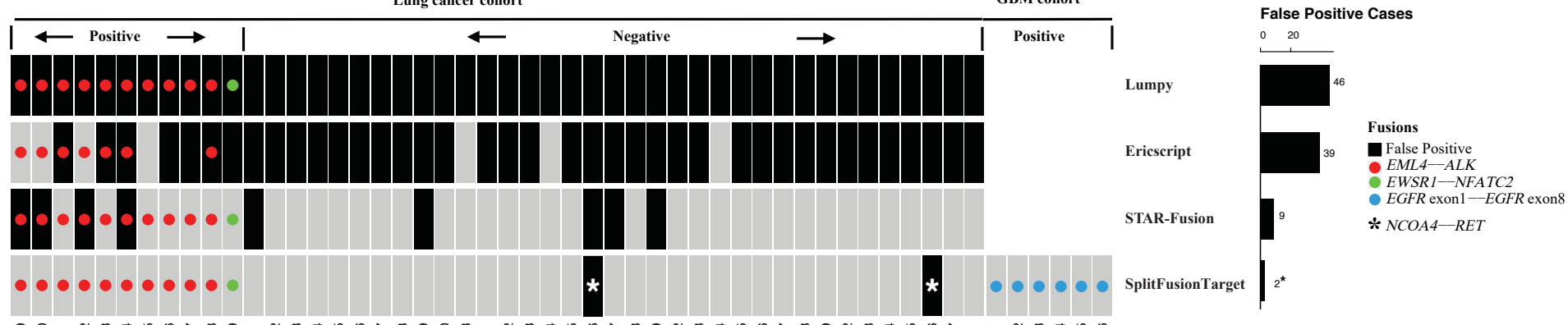

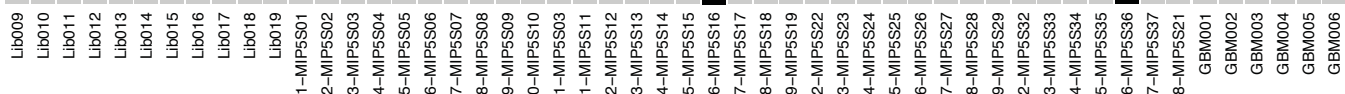

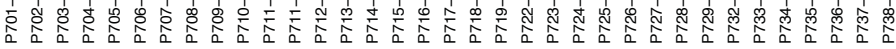

Figure 3. Fusion calling results for the 46 lung cancer samples with clinical known diagnoses (11 positive and $35 A L K-$ negative) by the four software - Ericscript, Lumpy, STAR-Fusion and SplitFusion. Each column represented one sample. Different colors and symbols denoted different fusions. Total number of false-positive calls (other than $A L K / E W S R 1$ ) for each software was plotted in the barplot. Out of 35 cases of glioblastoma (GBM), six were found with EGFR vIII using SplitFusion-Target. The GBM samples were not subjected for analysis by the other three software. 


\section{Expanded clinical analyses in 1,076 lung cancer FFPE samples}

215 To evaluate the robustness of SplitFusion performance in clinical samples, we expanded SplitFusion analyses on targeted sequencing of 1,076 clinical lung cancer (mean age 59.6 y, male 57.8\%) FFPE

217 samples using a 62-gene panel (Extended Data Tab. 2). SplitFusion detected 86 (8.0\%) fusionpositive cases in lung cancers (Fig. 4a). The prevalence of $A L K(4.65 \%)$, diverse partner-ROS1 $(1.95 \%)$ and diverse partner-RET $(1.12 \%)$ fusions were comparable to previously reported ${ }^{16}$. Rare oncogenic drivers and therapeutics relevant gene fusions KLC1-ALK, CD74-NRG1 and TPR-NTRK1 were also identified (Fig. 4a \& Extended Data Fig. 4). Interestingly, SplitFusion detected new fusions that have not been reported in lung cancer previously, including one FGFR3-JAKMP1 squamous cell carcinoma, one CLIP2-BRAF (two fusion isoforms) adenocarcinoma, and one ITPR2ETV6 adenocarcinoma (Fig. 4a \& Extended Data Fig. 5). We did not attempt to call MET exon 14skipping based on RNA-seq data alone, as the low-level RNA splicing donor and acceptor from nonconsecutive exons that are spatially proximal could form exon-skipping mRNAs, which are however not substantial when the cells divide and should be distinguished from the true cancer drivers root at the DNA level. Combined RNA exon-skipping and DNA splicing site mutations analyses simultaneous RNA and DNA target enrichment in wet lab and data analysis (e.g. using BWA for mapping both DNA and RNA reads) - await further developments.

Multiple gene fusion variants can co-occur in the same tumor ${ }^{15}$ and different variants may affect clinical outcomes ${ }^{18,27,28}$. However, the frequencies of different variant co-existences are largely unknown. Using SplitFusion, we found that multiple gene fusion variants with in-framed exons of partner genes, with or without involving cryptic splice site sequences, co-occurred frequently in the same tumors (Fig. 4b). Among the kinase genes analyzed, EML4-ALK v3 and CD74-ROS1 harboring lung tumors had the most frequent co-occurrences of multiple fusion variants.

Breakpoint-defined subclone

240 To dissect the intratumor clonal heterogeneity, fusion subclones were defined by the exact

241 breakpoints inferred from genomic mapping of fusion RNA sequences (Fig. 5a). Our results showed 
a
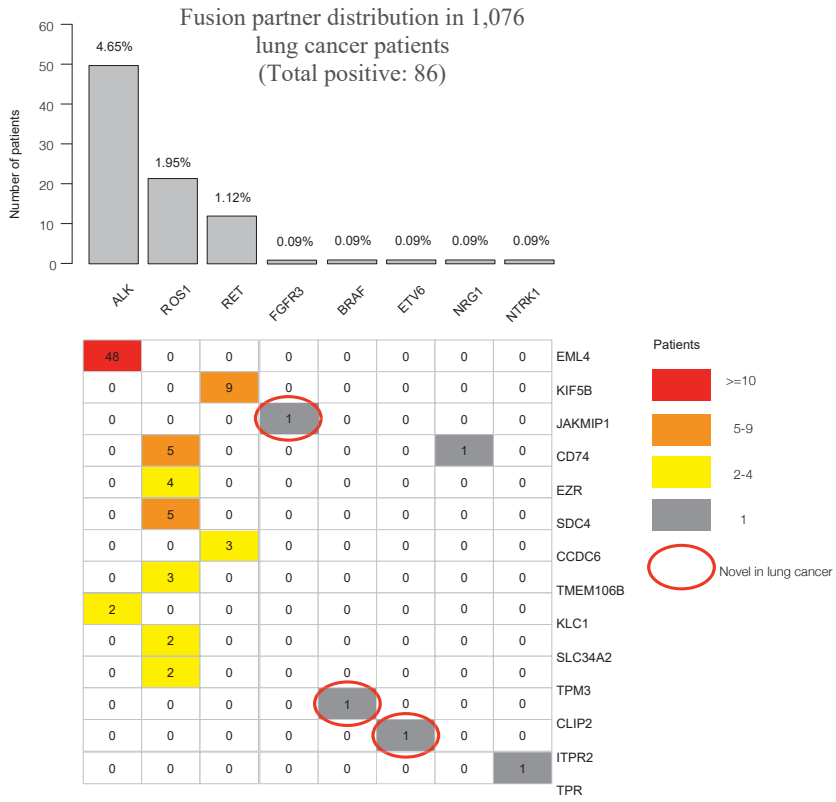

b

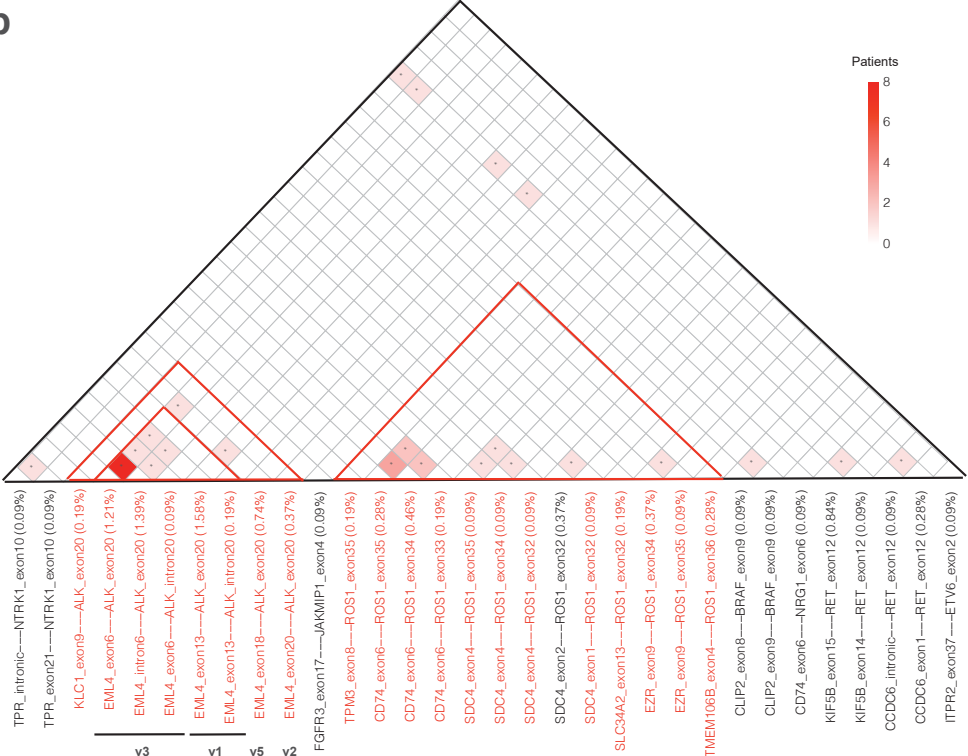

Figure 4. The analysis of 1,076 clinical lung cancer formalin-fixed paraffin-embedded samples using SplitFusion. (a) The prevalence of different target gene fusions were shown in the barplot. In total, 86 (8.0\%) samples were fusion-positive. The numbers of target genes with partner genes (right column) were indicated in the cells. (b) The numbers of co-occurrence of fusions in the same samples were plotted in the matrix. The EML4-ALK fusion variants were denoted under each specific fusion. 
EML4 exon20 - ALK exon20 (v2) EML4 exon18 - ALK exon20 (v5) $E M L 4$ exon13 - $A L K$ exon20 (v1) EML4 exon13 -- ALK intron20 (v1b) EML4 exon6 - ALK intron20 (v3a) EML4 exon6 - ALK exon20 (v3b) EML4 intron6 - ALK exon20 (v3c) EML4 intron6 - $A L K$ exon20 (v3d) EML4 intron6 - $A L K$ exon20 (v3e) $E M L 4$ intron6 - $A L K$ exon20 (v3f) b

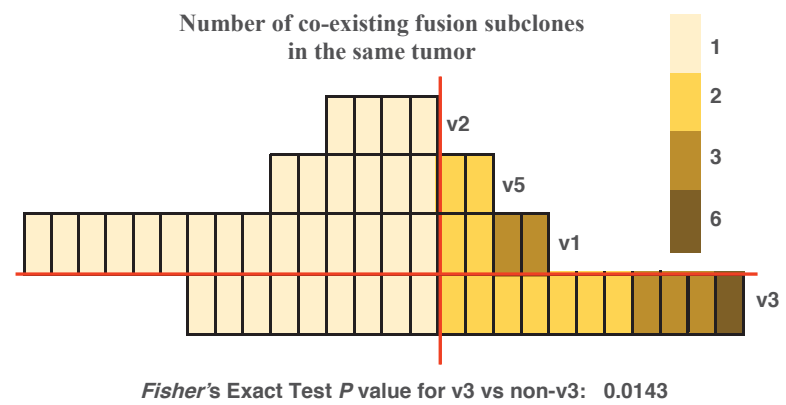

Fisher's Exact Test $P$ value for v3 vs non-v3: 0.0143

c

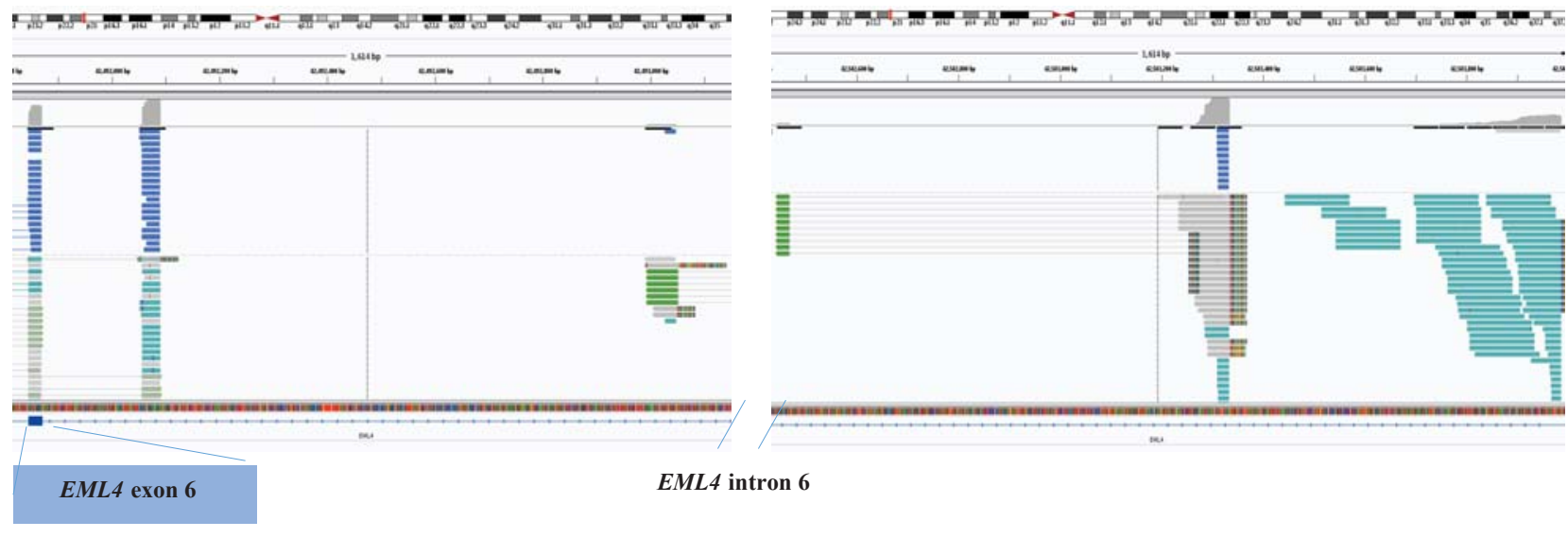

Figure 5. The analysis of breakpoint-defined subclones in $E M L 4-A L K$ positive lung tumors. (a) The schematic representations of different $E M L 4-A L K$ fusion variants. Blue cells represent $E M L 4$ exons and yellow cells represent $A L K$ exons. Numbers inside the cells showed exon numbers. Cells with red strokes represents introns. (b) Each tile represents one patient. The tile color gradients denote the number of co-existing fusion subclones, defined by exact breakpoint, in the same tumor. (c) For one $E M L 4-A L K$ variant 3 lung tumor sample, the Integrated Genome Viewer (IGV) showed 6 fusion breakpoints in $E M L 4$ exon 6 and intron 6. Their gene partner $A L K$ exon 20 was schematically shown on the right in red, and with nested gene specific primers 1 and 2. 
that $55 \%$ (11 of 20) of EML4-ALK v3 tumors had co-existing heterogeneous fusion subclones in the

243 same tumors (7 two-subclone, 3 three-subclone, and 1 six-subclone; Fig. 5 b \& c), which was

244 significantly higher than 19\% (6 of 31) in other variant tumors (4 of 19v1, 2 of 8 v5 and none of 4 v2;

245 Fisher's Exact Test two-sided $P$ value $=0.0143$ ).

\section{Discussion}

248 We demonstrate that SplitFusion is fast, highly sensitive, specific and computationally efficient in the

249 detection of gene fusions. SplitFusion can infer fusion frame-ness and exon-boundary alignment,

250 which are particularly desirable in the clinical setting so as to minimize non-trivial filtering of false-

251 positive or functionally insignificant fusion candidates. SplitFusion-Target mode allows for continuous evidence-based improvement in clinical reporting. Furthermore, SplitFusion is agnostic to known coding transcripts and can profile multiple co-existing fusion subclones, providing a powerful tool for research studies.

In a previous matched tissue-blood analysis, $21 \%$ of ALK-positive patients showed different EML4$A L K$ variants between tissue and blood samples ${ }^{20}$, indicating the existence of fusion-defined tumor heterogeneity evident at the genomic level. SplitFusion can comprehensively map cryptic splice site breakpoints and has identified up to six subclones co-existing in one EML4-ALK v3 tumor, with different breakpoints in EML4 intron 6. Increasing evidences have suggested that lung cancer patients with EML4-ALK v3 tumors may have less favorable clinical outcomes compared to patients with other EML4-ALK variants ${ }^{18,19,29}$. The most frequent resistance mutation $A L K$ G1202R was shown to be present exclusively in the v3 of EMLA-ALK tumors ${ }^{18}$. Heuckmann et al. showed that the v3 protein had a higher stability than other variant proteins ${ }^{30}$. However, the expression of the v3 cDNA (homogenous) in $\mathrm{Ba} / \mathrm{F} 3$ cells precluded the study of $\mathrm{v} 3$ subclones (heterogeneous) that may present in clinical tumors. When using clinical tumor derived cell lines, the study of ALK inhibitor on the v1harbouring cell line H3122 showed the typical monotonic S-shape dose-response relationship (homogeneous) $^{31}$. In contrast, inhibition of the v3b-harbouring cell line H2228 was observed across a wide range of ALK inhibitor concentration ${ }^{31}$, indicating heterogeneous responses among the $\mathrm{H} 2228$ 
cell population. Insertions (in-framed) could lead to more profound protein structure and property

271 changes than do point mutations ${ }^{32}$. Therefore, $\mathrm{v} 3 \mathrm{a} / \mathrm{b}(\mathrm{c} / \mathrm{d} / \mathrm{e} / \mathrm{f})$-harboring tumors may contain

272 structurally diverse ALK proteins and easier to develop resistances to a single targeting agent. Further studies are needed to investigate whether EML4 intron 6 is a breakpoint 'hot-zone' that may provide a high genetic propensity for evolving resistance clones along the course of targeted therapy. Among ROS1-positive lung cancers, we also observed that, albeit a small number, CD74-ROS1 tumors had more fusion subclones than ROS1 tumors with other fusion partners. Interestingly, one recent study

277 showed that lung cancer patients with CD74-ROS1 had a lower survival rate compared to those with other ROS1 partners ${ }^{33}$. Our finding suggests that fusion-defined subclone analyses may be important in refining diagnosis and treatment for fusion-positive tumors in the future.

280

Limitations of SplitFusion includes the inability to detect non-reference sequences as fusion partners, such as from viruses. In this case, one can combine human and suspected viral genome FASTA files, and use BWA ('bwa index') to build a combo reference genome. Our observation of the breakpoint 'hot-zone' in EML4 intron 6 needs to be confirmed by larger studies. Further, whether this phenomenon could explain the resistances in the targeted treatment of the EMLA-ALK v3 tumors requires further mechanism studies and prospective clinical investigations. In conclusion, SplitFusion is fast, sensitive, specific and well-suited for broad use in clinics for gene fusion detection, as well as in research for studying fusion-defined tumor heterogeneity.

\section{Methods}

Performance benchmarking with other software

We selected three other fusion detection software to benchmark the performance of SplitFusion for several reasons. In a recent comprehensive comparison of 23 different software, STAR-Fusion showed the highest accuracy (ranked \#1) and was among the fastest software ${ }^{14}$. EricScript ${ }^{12}$ was topranked among 12 well-known software in an earlier study ${ }^{34}$. Lumpy was not among the software compared by the aforementioned two studies, but was able to integrate read-pair, split-read and readdepth jointly ${ }^{13}$, especially when structure variation (fusion) signal is reduced owing to either low 
coverage data or low intra-sample variant (fusion) allele frequency, which are common in cancer samples.

300

301 Performance of the four software was compared using 46 RNA sequencing datasets generated from clinical FFPE samples (11 positive and 35 negative cancer cases; see Clinical next-generation sequencing for details). For the 11 positive sample datasets, each was randomly down-sampled using the seqtk tool (v1.0-r31; https://github.com/lh3/seqtk) to eight different sizes (3M, 1M, 500K, 100K, $50 \mathrm{~K}, 10 \mathrm{~K}, 7.5 \mathrm{~K}$, and $5 \mathrm{~K}$ reads), each size had 21 replicates, generating a total of 1,848 datasets for benchmarking test. All of the software were run with one computing thread using human genome GRCh37-hg19 as the reference, and fusion analyses were run using default parameters (e.g. the default threshold for STAR-Fusion to call a fusion is one split-read ['min_junction_reads' default: 1]).

The performance among the four software was scored based on the following metrics: 1) Sensitivity: the fraction of samples with the correct fusion genes (ALK and EWSRI) detected among the 11

312 fusion-positive samples. 2) Unconfirmed or nonsignificant fusions: fusions involving genes other than the expected $A L K$ and EWSR1.3) AUC: the area under the precision-recall curve at varied supporting read thresholds (from minimum to maximum supporting reads) according to the previous method ${ }^{14} .4$ ) Peak memory: the maximum resident set size (RSS: the portion of memory occupied by a process that is held in main memory) during the computation of the software. 5) User time: the amount of CPU time for a given analysis. Publically available database and tool versions used: human genome reference hg19, LUMPY v0.2.13, EricScript v2.1, STAR-Fusion v1.5, bwa v0.7.17, Samtools v1.10, BEDTools-v2.27.1, seqtk v1.0-r31, ANNOVAR (2019Oct24), PERL v5, R v3.6.3, Python v2.7/v3.

\section{Clinical next-generation sequencing}

322 In this study, $10 A L K$-positive lung cancer and one EWSRI-positive sarcoma ${ }^{35}$ samples, confirmed by clinical FISH assays, and 35 ALK FISH-negative lung cancer samples from Queen Elizabeth Hospital,

324 Hong Kong, were included. The study was approved by the Research Ethics Committee, Hospital 325 Authority, Hong Kong (KC/KE-16-0262/ER-1), institutional review boards at Zhejiang Cancer 
327 (TNA) containing RNA and DNA were extracted from FFPE sections using the Agencourt FormaPure

328 Kit for FFPE Tissue (Beckman Coulter, Indianapolis, IN). NGS libraries were constructed using the

329 anchored multiplex PCR targeted RNA-seq method described previously ${ }^{15}$, with modifications for

330 incorporating unique molecular identifier (UMI) in the adaptors and using an one-tube assay for

331 simultaneous enrichment of RNA and DNA targets ${ }^{36}$. Briefly, 100 ng of TNA was used for reverse

332 transcription by SuperScript IV (ThermoFisher) using random hexamers, followed by second strand

333 synthesis. The double strand cDNAs were purified using 1.8X SPRIselect (Beckman Coulter) and

334 subjected to further library construction using the KAPA HyperPlus Kit (Roche). The purified ds-

335 cDNAs were enzymatically fragmented at $37^{\circ} \mathrm{C}$ for $15 \mathrm{~min}$, then end-repaired and A-tailed to produce

336 5'-phosphorylated and 3'-dA-tailed by incubating with the End-repair \& A-tailing enzyme at $37^{\circ} \mathrm{C}$ for

$33715 \mathrm{~min}$, followed by $65^{\circ} \mathrm{C}$ for $15 \mathrm{~min}$. Adapters containing sample index and $\mathrm{UMI}^{37}$ were then ligated

338 to the End-repaired and A-tailed DNA fragments using DNA ligase with 25 pmol of annealed

339 adapters at $16^{\circ} \mathrm{C}$ for $30 \mathrm{~min}$, followed by $22^{\circ} \mathrm{C}$ for $30 \mathrm{~min}$. Post-ligation cleanup was performed using

340 1.0X SPRIselect (Beckman Coulter). The size-selected ligation products were eluted in $20 \mu \mathrm{L}$

341 nuclease-free water and amplified by two rounds of hemi-nested PCRs for target enrichment. In

342 PCR1, the ligated DNA fragments were added to $3 \mu \mathrm{L}$ of $10 \mathrm{X}$ PCR buffer, a final concentration of 2

$343 \mathrm{mM}$ of $\mathrm{MgCl}_{2}, 0.2 \mathrm{mM}$ of dNTP (New England Biolabs), 25 pmol of panel-specific GSP1 enrichment

344 primers, 3 Units of Platinum ${ }^{\mathrm{TM}}$ Taq DNA polymerase (Invitrogen) and topped up to a final volume of

$34530 \mu \mathrm{L}$ of nuclease-free water. The thermo cycling conditions were as follows: initial denaturation at

$34695^{\circ} \mathrm{C}$ for $5 \mathrm{~min}$; followed by 20 cycles of $95^{\circ} \mathrm{C}$ for $30 \mathrm{sec}$ and ramping down at $-0.2^{\circ} \mathrm{C} / \mathrm{sec}$ to $60^{\circ} \mathrm{C}$ for

$3471 \mathrm{~min}$; hold at $4^{\circ} \mathrm{C}$. The amplified PCR1 products were washed with 1.2 X SPRIselect and eluted in

$34820 \mu \mathrm{L}$ nuclease-free water. In PCR2, $20 \mu \mathrm{L}$ of the purified PCR1 products were mixed with $3 \mu \mathrm{L}$ of

349 10X PCR buffer, a final concentration of $2 \mathrm{mM}$ of $\mathrm{MgCl}_{2}, 0.2 \mathrm{mM}$ of dNTP, 25 pmol of panel-

350 specific GSP2 enrichment primers, 10 pmol of P5 primer, 10 pmol of P7 indexing primer ${ }^{37}, 3$ Units of

351 Platinum $^{\mathrm{TM}}$ Taq DNA Polymerase and topped up to $30 \mu \mathrm{L}$ with nuclease free water. The thermo

352 cycling parameters were as follows: initial denaturation at $95^{\circ} \mathrm{C}$ for $5 \mathrm{~min}$; followed by 15 cycles of

$35395^{\circ} \mathrm{C}$ for $30 \mathrm{sec}$ and $65^{\circ} \mathrm{C}$ for $5 \mathrm{~min}$; hold at $4^{\circ} \mathrm{C}$. The final library constructs were size selected by 0.7 
X SPRIselect and eluted in $30 \mu \mathrm{L}$ of EB buffer. After quantification by KAPA Library Quantification

355 Kit for Illumina (Roche), the constructed libraries were subsequently pooled and sequenced by the Illumina NextSeq 500 System. Similarly, the lung cancer and glioblastoma cohort samples were analyzed as previously described ${ }^{38}$.

\section{Implementation and availability.}

360 The code implementing all steps is included in the SplitFusion software package. SplitFusion is available on GitHub (https://github.com/Zheng-NGS-Lab/SplitFusion). User manual and example datasets are also included. The datasets generated during and analyzed during the current study are available from the corresponding author on reasonable request.

364

\section{Acknowledgments}

We thank financial supports from Ming Wai Lau Centre of Reparative Medicine of Karolinska Institutet (Lau Grant), City University of Hong Kong (internal grant), the National Natural Science Foundation of China (81672098 to Z.Z.; 81872072 to J.C.), The Hong Kong Research Grants Council (11319516 to M.Y. and Z.Z.), The Innovation and Technology Fund of Hong Kong Government (9440153 to Z.Z.), and The Swedish Research Council (2016-02830 to Z.Z.).

\section{Author contributions}

B.Z. and C.B. performed bioinformatics analyses and packaged the pipeline; Z.S., C.X., W.W., C.L. and Y.Z. designed and analyzed the large lung cancer cohort; A.H.Y.C performed bioinformatics analysis using benchmarking software; H.W., Z.G., and J.C. designed and analyzed the glioblastoma cohort; C.L., S.B., H.Y.K. and M.Z.C. performed NGS experiments; W.C., M.Y., and W.C.S.C designed and analyzed the initial clinical cohort study; M.Y., W.C.S.C., J.C. and Z.Z. supervised the research and obtained funding; Z.Z. designed SplitFusion; B.Z. and Z.Z. wrote the paper; all authors approved the paper.

\section{Competing interests}


383 Hospital. Z.Z. and M.Y. are scientific advisors for and hold equity in Helitec, which interests are

reviewed and regulated by institutional Outside Practice and Outside Work policies annually.

385

386

387

388

389

390

391

392

393

394

395

396

397

398

399

400

401

402

403

404

405

406

407

408

409

410

411

412

413

414

415

416

417

418

419

420

421

422

423

424

425

426

427

428

429

430

431

432

433

\section{References}

1. Shaw, A. T., Hsu, P. P., Awad, M. M. \& Engelman, J. A. Tyrosine kinase gene rearrangements in epithelial malignancies. Nat. Rev. Cancer 13, 772-87 (2013).

2. Druker, B. J. et al. Efficacy and Safety of a Specific Inhibitor of the BCR-ABL Tyrosine Kinase in Chronic Myeloid Leukemia. N. Engl. J. Med. 344, 1031-1037 (2001).

3. Shen, Z. X. et al. All-trans retinoic acid/AS2O3 combination yields a high quality remission and survival in newly diagnosed acute promyelocytic leukemia. Proc. Natl. Acad. Sci. U. S. A. 101, 5328-5335 (2004).

4. Kwak, E. L. et al. Anaplastic Lymphoma Kinase Inhibition in Non-Small-Cell Lung Cancer. N. Engl. J. Med. 363, 1693-1703 (2010).

5. Shaw, A. T. et al. Crizotinib in ROS1-rearranged non-small-cell lung cancer. N. Engl. J. Med. 371, 1963-1971 (2014).

6. Tomlins, S. A. Recurrent Fusion of TMPRSS2 and ETS Transcription Factor Genes in Prostate Cancer. Science. 310, 644-648 (2005).

7. Wu, Y.-M. et al. Identification of targetable FGFR gene fusions in diverse cancers. Cancer Discov. 3, 636-47 (2013).

8. Mullard, A. FDA approves landmark tissue-agnostic cancer drug. Nat. Rev. Drug Discov. 18, 7 (2018).

9. Gao, Q. et al. Driver Fusions and Their Implications in the Development and Treatment of Human Cancers. Cell Rep. 23, 227-238.e3 (2018).

10. Stransky, N., Cerami, E., Schalm, S., Kim, J. L. \& Lengauer, C. The landscape of kinase fusions in cancer. Nat Commun 5, 4846 (2014).

11. Gan, H. K., Cvrljevic, A. N. \& Johns, T. G. The epidermal growth factor receptor variant III (EGFRvIII): where wild things are altered. FEBS J. 280, 5350-5370 (2013).

12. Benelli, M. et al. Discovering chimeric transcripts in paired-end RNA-seq data by using EricScript. Bioinformatics 28, 3232-9 (2012).

13. Layer, R. M., Chiang, C., Quinlan, A. R. \& Hall, I. M. LUMPY: a probabilistic framework for structural variant discovery. Genome Biol. 15, R84 (2014).

14. Haas, B. J. et al. Accuracy assessment of fusion transcript detection via read-mapping and de novo fusion transcript assembly-based methods. Genome Biol. 20, 213 (2019).

15. Zheng, Z. et al. Anchored multiplex PCR for targeted next-generation sequencing. Nat. Med. 20, 1479-1484 (2014).

16. Zehir, A. et al. Mutational landscape of metastatic cancer revealed from prospective clinical sequencing of 10,000 patients. Nat. Med. 23, 703-713 (2017).

17. Li, H. Aligning sequence reads, clone sequences and assembly contigs with BWA-MEM. arXiv Prepr. arXiv1303.3997 (2013).

18. Lin, J. J. et al. Impact of EML4-ALK Variant on Resistance Mechanisms and Clinical Outcomes in ALK -Positive Lung Cancer. J. Clin. Oncol. 36, 1199-1206 (2018).

19. Christopoulos, P., Kirchner, M., Endris, V., Stenzinger, A. \& Thomas, M. EML4-ALK V3, treatment resistance, and survival: Refining the diagnosis of ALK+ NSCLC. Journal of Thoracic Disease 10, S1989-S1991 (2018).

20. Camidge, D. R. et al. Updated Efficacy and Safety Data and Impact of the EML4-ALK Fusion Variant on the Efficacy of Alectinib in Untreated ALK-Positive Advanced Non-Small Cell Lung Cancer in the Global Phase III ALEX Study. J. Thorac. Oncol. 14, 1233-1243 (2019).

21. Quinlan, A. R. \& Hall, I. M. BEDTools: a flexible suite of utilities for comparing genomic features. Bioinformatics 26, 841-842 (2010).

22. Kent, W. J. BLAT---The BLAST-Like Alignment Tool. Genome Res. 12, 656-664 (2002).

23. Mount S M. A catalogue of splice junction sequences. Nucleic Acids Res. 10, 459-472 (1982). 
24. Wang, K., Li, M. \& Hakonarson, H. ANNOVAR: functional annotation of genetic variants from high-throughput sequencing data. doi:10.1093/nar/gkq603

25. Cingolani, P. et al. A program for annotating and predicting the effects of single nucleotide polymorphisms, SnpEff: SNPs in the genome of Drosophila melanogaster strain w1118; iso-2; iso-3. Fly (Austin). 6, 80-92 (2012).

26. Pruitt, K. D., Tatusova, T. \& Maglott, D. R. NCBI Reference Sequence (RefSeq): A curated non-redundant sequence database of genomes, transcripts and proteins. Nucleic Acids Res. 33, (2005).

27. Yoshida, T. et al. Differential crizotinib response duration among ALK fusion variants in ALK-positive non-small-cell lung cancer. J. Clin. Oncol. 34, 3383-3389 (2016).

28. Lin, J. J. \& Shaw, A. T. Differential Sensitivity to Crizotinib: Does EML4-ALK Fusion Variant Matter? J. Clin. Oncol. 34, 3363-5 (2016).

29. Christopoulos, P. et al. EML4-ALK fusion variant V3 is a high-risk feature conferring accelerated metastatic spread, early treatment failure and worse overall survival in ALK+ nonsmall cell lung cancer. Int. J. Cancer 142, 2589-2598 (2018).

30. Heuckmann, J. M. et al. Differential Protein Stability and ALK Inhibitor Sensitivity of EML4ALK Fusion Variants. Clin. Cancer Res. 18, 4682-4690 (2012).

31. Woo, C. G. et al. Differential protein stability and clinical responses of EML4-ALK fusion variants to various ALK inhibitors in advanced ALK-rearranged non-small cell lung cancer. Ann. Oncol. 28, 791-797 (2017).

32. Vetter, I. R. et al. Protein structural plasticity exemplified by insertion and deletion mutants in T4 lysozyme. Protein Sci. 5, 2399-2415 (1996).

33. Li, Z. et al. Efficacy of Crizotinib among Different Types of ROS1 Fusion Partners in Patients with ROS1-Rearranged Non-Small Cell Lung Cancer. J. Thorac. Oncol. 13, 987-995 (2018).

34. Kumar, S., Vo, A. D., Qin, F. \& Li, H. Comparative assessment of methods for the fusion transcripts detection from RNA-Seq data. Sci. Rep. 6, 1-10 (2016).

35. Yau, D. T. W. et al. Bone Sarcoma With EWSR1-NFATC2 Fusion: Sarcoma With Varied Morphology and Amplification of Fusion Gene Distinct From Ewing Sarcoma. Int. J. Surg. Pathol. 27, 561-567 (2019).

36. Zheng, Z. et al. Abstract 4895: A one-tube assay for simultaneous detection of gene mutation, fusion, copy number alteration and expression. in Cancer Research 75, 4895-4895 (American Association for Cancer Research (AACR), 2015).

37. Tan, Y. et al. Rationally engineered Staphylococcus aureus Cas9 nucleases with high genome-wide specificity . Proc. Natl. Acad. Sci. 201906843 (2019). doi:10.1073/pnas.1906843116

38. Song, Z. et al. Simultaneous Detection of Gene Fusions and Base Mutations in Cancer Tissue Biopsies by Sequencing Dual Nucleic Acid Templates in Unified Reaction. Clin. Chem. 66, 178-187 (2020). 


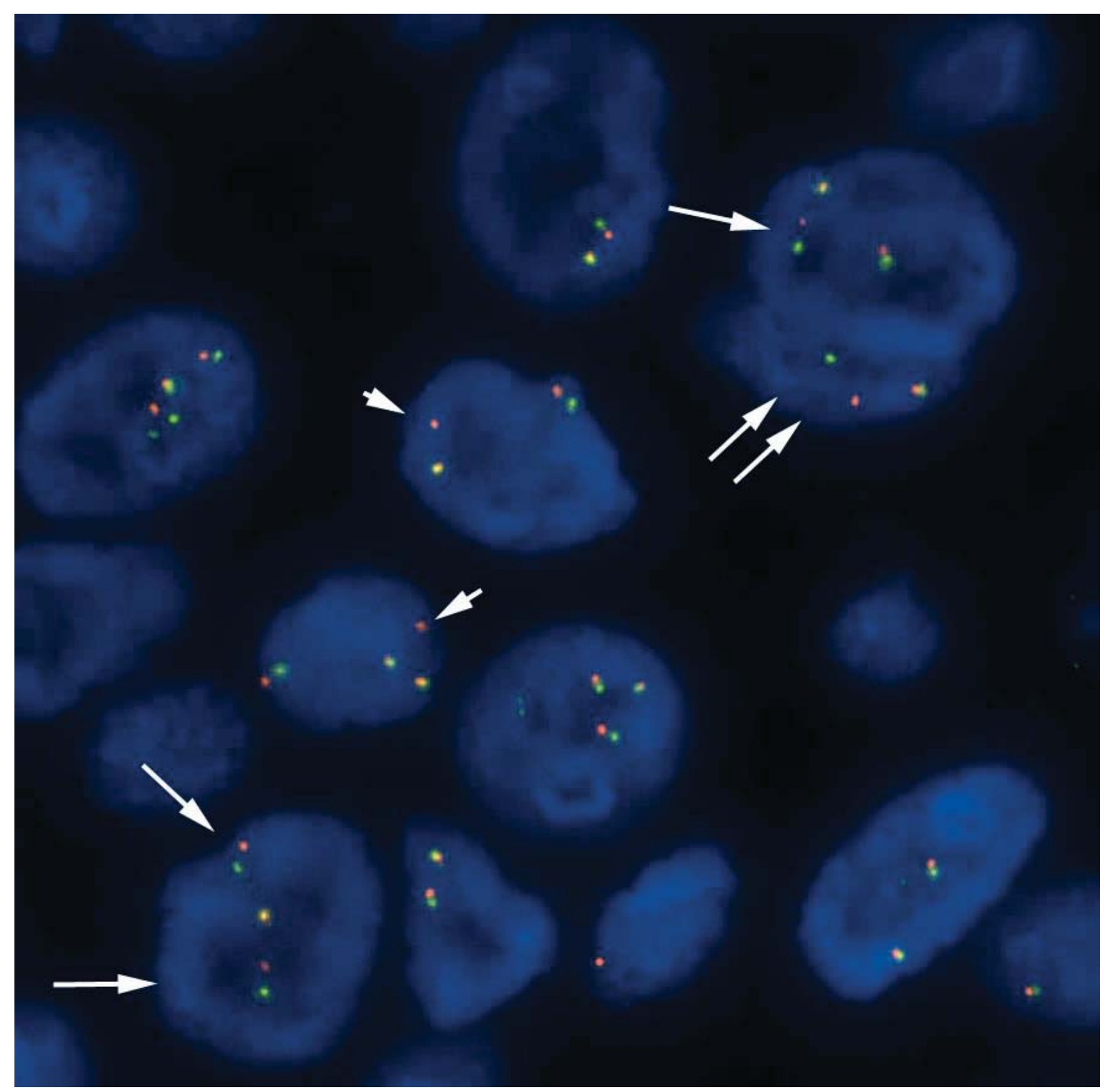

Extended Data Figure 1. Fluorescence in situ hybridization (FISH) performed on a lung cancer formalin-fix and paraffin-embedded sample using Vysis ALK breakapart probes. The result indicated the presence of $A L K$ gene translocation: separation of 3' (red) and 5' (green) signals were indicated by double arrows; narrow split of signals indicated by single arrows; isolated 3' signals indicated by short arrows. 


\section{P736-MIP5S36.NCOA4_exon4---RET_exon13}

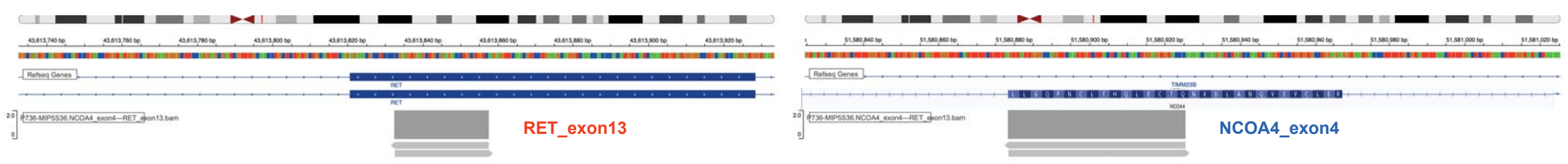

P716-MIP5S16.NCOA4_exon4---RET_exon13

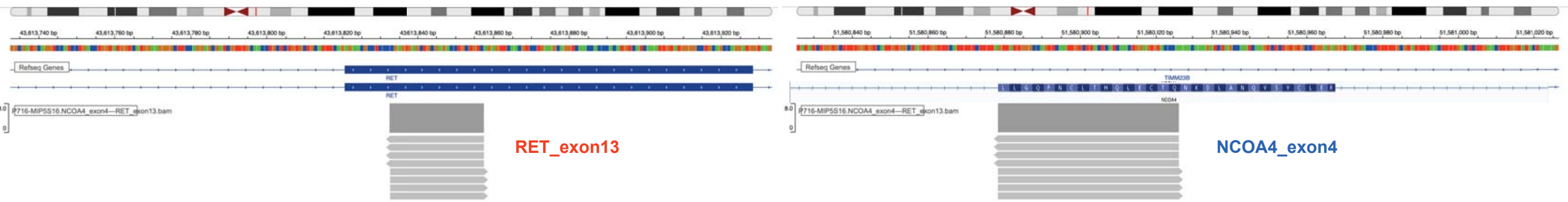

Extended Data Figure 2. Visualization of NCOA4-RET gene fusions showed the alignment of detected partner gene NCOA4 (colored in blue) on exon boundary. 
EGFR exon1 --- EGFR exon8

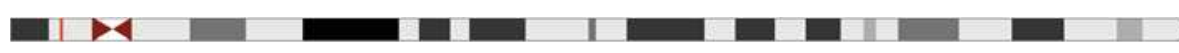

\begin{tabular}{lllllllll}
$55,086,925$ bp & $55,086,950$ bp & $55,086,975$ bp & $55,087,000$ bp & $55,087,025$ bp & $55,087,050$ bp & $55,087,075$ bp & $55,087,100$ bp & 55 \\
\hline
\end{tabular}

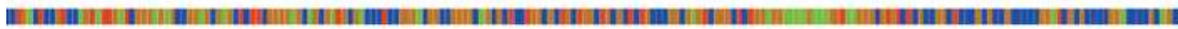
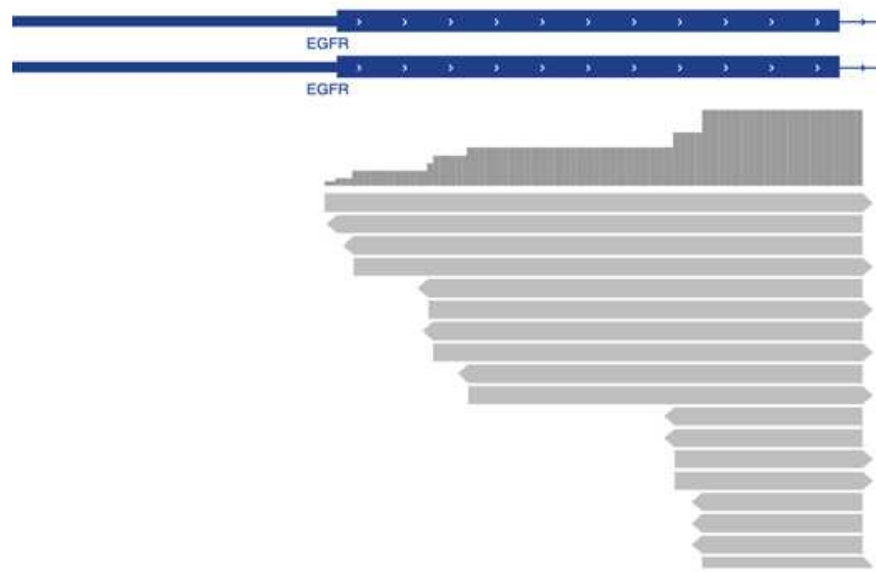

EGFR exon1

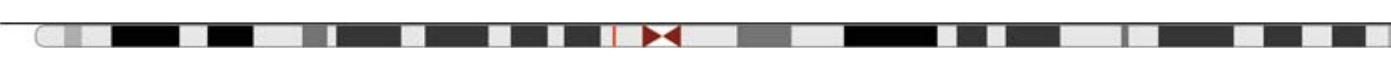

$\begin{array}{llllllllll}55,223,450 \mathrm{bp} & 55,223,475 \mathrm{bp} & 55,223,500 \mathrm{bp} & 55,223,525 \mathrm{bp} & 55,223,550 \mathrm{bp} & 55,223,575 \mathrm{bp} & 55,223,600 \mathrm{bp} & 55,223,625 \mathrm{bp} & 55,223,650 \mathrm{bp} & 55,223,675\end{array}$ แตห Refseq Genes.

$\int_{0}^{20}$ 19-10729T.EGFR_exon1--EGFR_exon8.bam
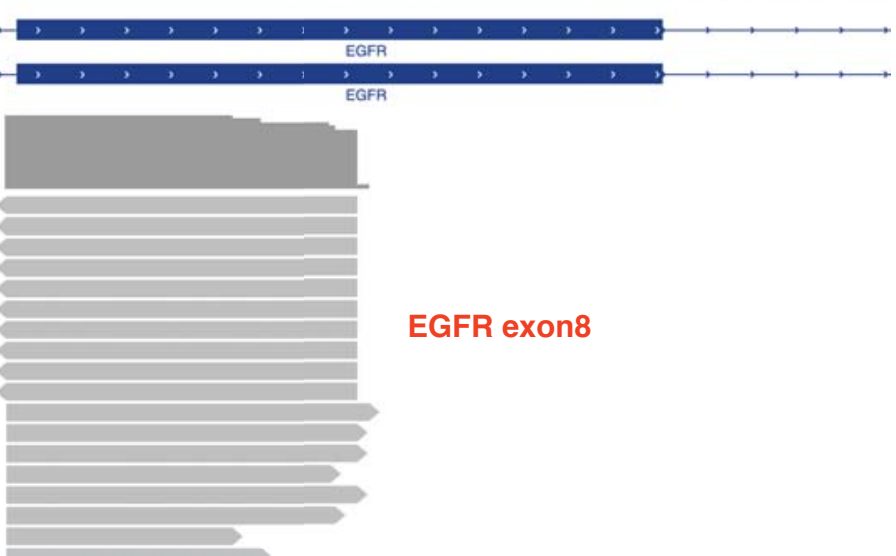

EGFR exon8

Extended Data Figure 3. Visualization of EGFR $v$ III showed the transcript with exons 2 - 7 deletion in a glioblastoma sample (targeted exon 8 colored in red and detected parter exon 1 in blue). 
KLC1_exon9---ALK_exon20

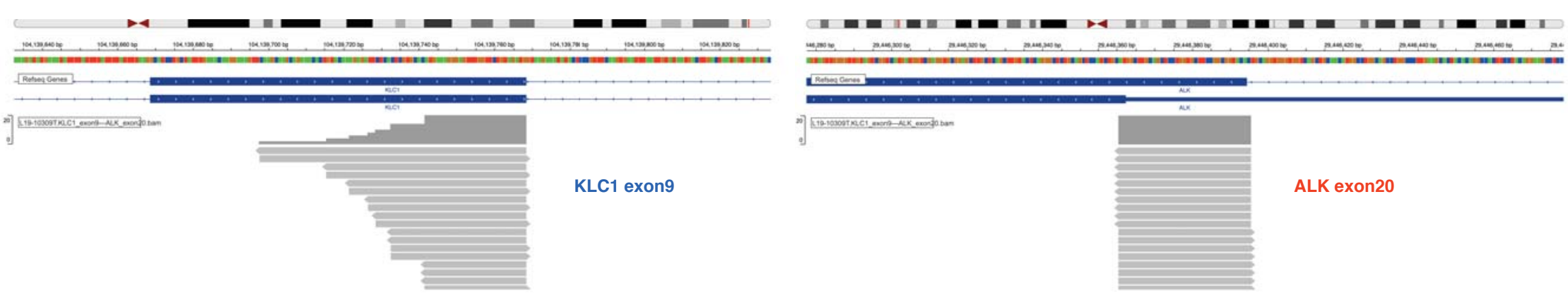

CD74_exon6---NRG1_exon6

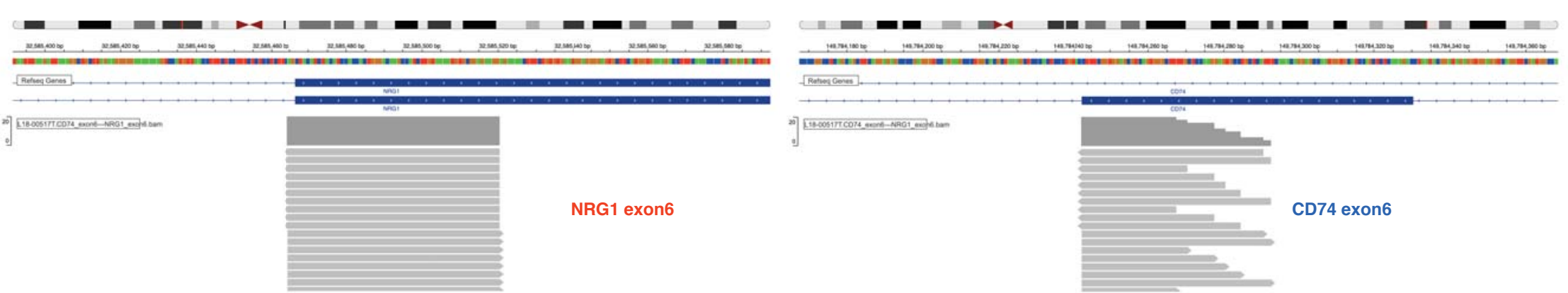

TPR_exon21---NTRK1_exon10

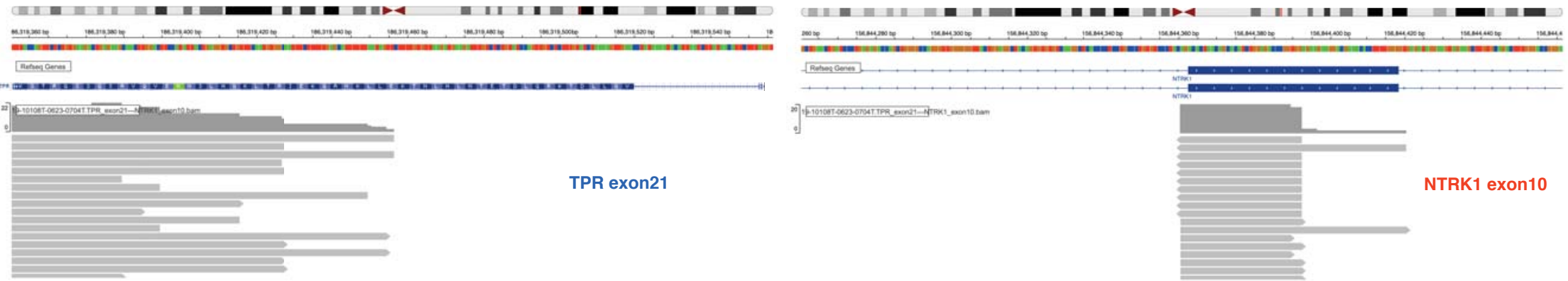

Extended Data Figure 4. Visualization of rare fusions in lung cancer showed typical anchored multiplex PCR detected fusions involving targeted genes (colored in red; rectangle pileup) and detected fusion partners (colored in blue; open-end pileup) for KLC1-ALK, CD74-NRG1, and TPR-NTRK1. All of the fusion partners showed the alignments on exon boundary. 
>FGFR3 exon 17 --- JAKMIP1 exon 4

GACCGTGTCCTTACCGTGACGTCCACCGACATGGATGAGATCAAAGGGAAAGACCGTGTGATTCTGGCCTTGGAGAAGGAACTTGGCGTGCAGGCTGGGCAGACC

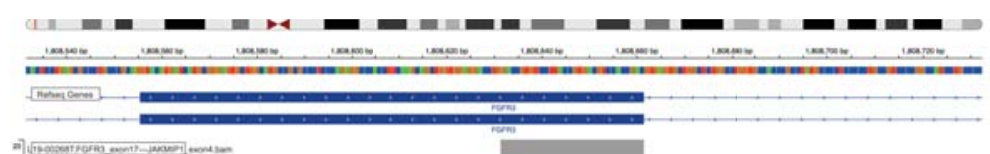

in

FGFR3 exon17

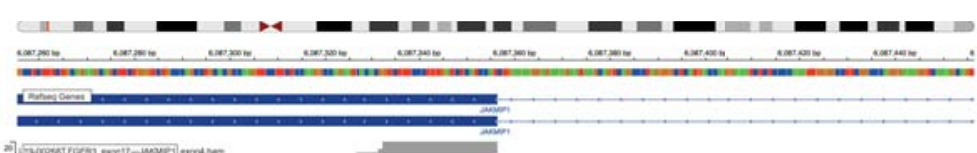

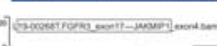

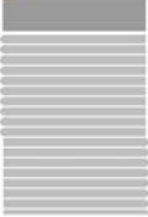

JAKMIP1 exon4

$>$ CLIP2 exon 9 --- BRAF exon 9

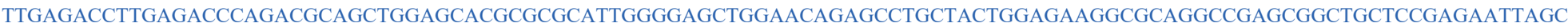
GGACAACAGGGACTTGATTAGAGACCAAGGATTTCGTGGTGA

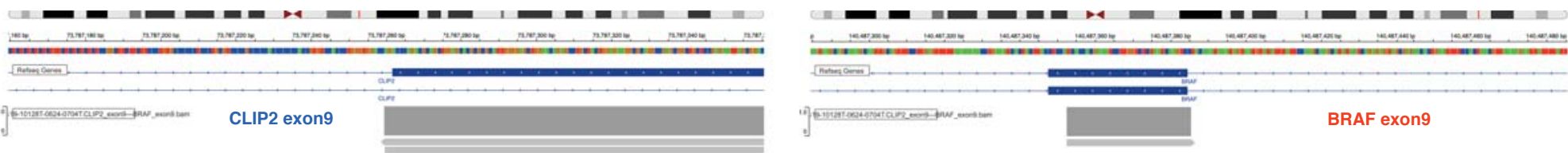

>CLIP2 exon 8 --- BRAF exon 9

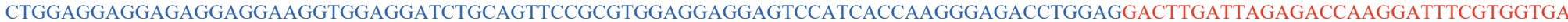
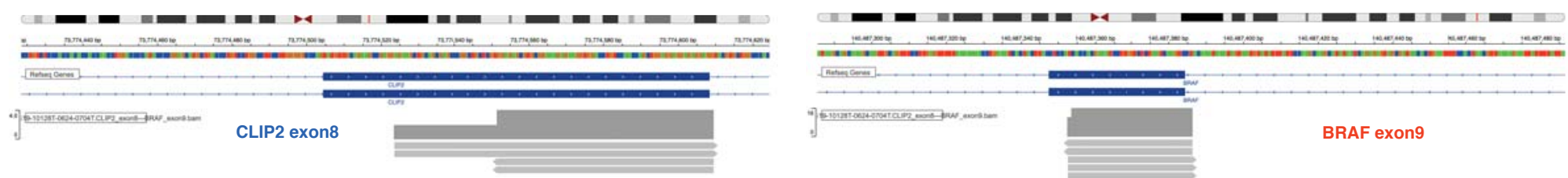

>ETV6 exon 2 --- ITPR2 exon 37

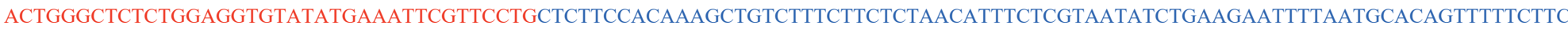
TTTCTCCATTAGTTTCTTTGTATGATTAATCAACTTC

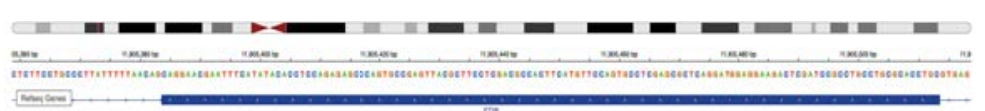

i)

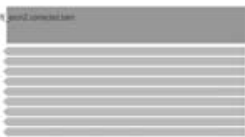

ETV6 exon2

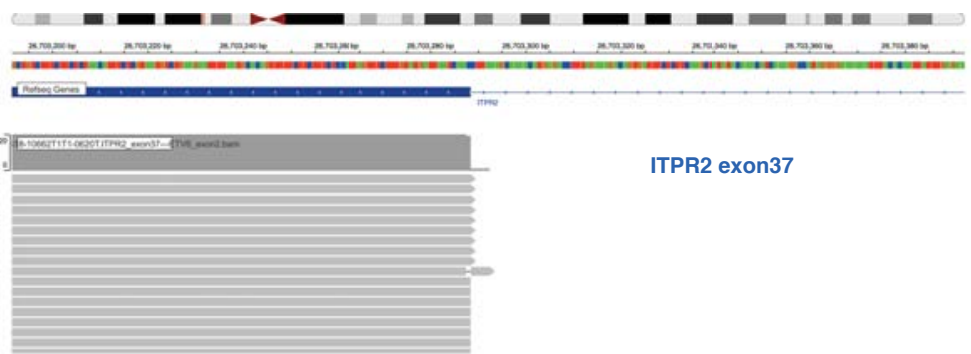

Extended Data Figure 5. Visualization of new fusions in lung cancer showed typical anchored multiplex PCR detected fusions involving targeted genes (colored in red; rectangle pileup) and detected fusion partners (colored in blue; open-end pileup) for FGFR3-JAKMIP1, CLIP2-BRAF, and ETV6-ITPR2. All fusion partners showed the alignments on exon boundary. 
Table 1. The list of RNA and DNA targets in a 19-gene lung cancer panel.

RNA targets

\begin{tabular}{llll}
\hline Gene & NM & Exon & $\begin{array}{l}\text { Sense } \\
\text { antisense }\end{array}$ \\
\hline ALK & NM_004304 & $19,20,21,22$ & antisense \\
EGFR & NM_005228 & $1,8,9$ & antisense \\
FGR & NM_005248 & $1,2,3$ & antisense \\
MET & NM_000245 & $13,14,15$ & antisense \\
NRG1 & NM_004495 & $1,2,3,4,6$ & antisense \\
NTRK1 & NM_002529 & $8,10,11,12,13$, & antisense \\
& & 14,15 & \\
NTRK2 & NM_006180 & $11,12,13,14,1$ & antisense \\
NTRK3 & NM_002530 & $13,16,17$ & \\
ROS1 & NM_002944,15,16 & antisense \\
RET & NM_020630 & $5,36,3,37,10,11,12,1$ & antisense \\
\hline
\end{tabular}

DNA targets (Reference: hg19)

\begin{tabular}{|c|c|c|c|c|c|c|c|c|c|}
\hline Gene & NM & start & end & chr & Gene & NM & start & end & chr \\
\hline$\overline{\mathrm{ALK}}$ & NM_ & 648 & 747 & 2 & $\overline{\text { FGFR1 }}$ & $\overline{\mathrm{NM} \_0}$ & 38 & 38 & \\
\hline & A_ & & & 2 & A & NM_ & & & \\
\hline & 4 & 06 & 277 & 2 & DK & NM_0 & 97 & 10 & \\
\hline $\mathrm{H} 2$ & 251 & & & 2 & $\Delta$ & NM_00 & 73 & & \\
\hline $\mathrm{H} 2$ & M_O & & & 2 & S & NM_O & & & 12 \\
\hline $\mathrm{H} 2$ & 51 & & 14 & 2 & 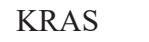 & M_O & 25 & & 12 \\
\hline & 1 & & & 2 & & 40 & & & 12 \\
\hline $\mathrm{H} 2$ & 1 & & & 2 & $\mathrm{~S}$ & M_C & & & 12 \\
\hline $\mathrm{H} 2$ & 4_0 & & & 2 & OLE & M_0C & 57 & 96 & 12 \\
\hline & $1 \_0$ & & & - & & M_0 & & & 12 \\
\hline $\mathrm{H} 2$ & 1_C & & & 2 & 53 & M_O & & & 17 \\
\hline $\mathrm{H} 2$ & {$\left[\begin{array}{l}0 \\
-\end{array}\right.$} & & & 2 & 53 & M_O & & & 17 \\
\hline $\mathrm{H} 2$ & 1 & & & 2 & & & & & 17 \\
\hline $\mathrm{H} 2$ & 10 & & & 2 & 53 & 46 & & & 17 \\
\hline & 1 & & & 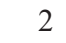 & & 0 & & & 17 \\
\hline $\mathrm{H} 2$ & I_ & & & 2 & & 6 & & & 17 \\
\hline & 1 & & & & & 1_ & & & 17 \\
\hline $\mathrm{H} 2$ & 1 & & & 2 & 53 & 6 & & & 17 \\
\hline $\mathrm{H} 2$ & 1 & & & 2 & & 6 & & & 17 \\
\hline $\mathrm{S} 1$ & I_O & & & & & M_O & & & 17 \\
\hline S1 & 4 & & & 6 & $\mathrm{RB}$ & NM_C & & & 17 \\
\hline S1 & I 0 & & & & 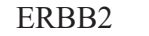 & M_O & & & 17 \\
\hline FR & 8 & & & & BBR2 & NM_0 & & & 17 \\
\hline FR & $1 \_0$ & & & 7 & 2 & M_O & & & 17 \\
\hline GFR & I $\_0$ & & & & IAD4 & NM_0 & & & 18 \\
\hline GFR & 40 & & & & $40 \mathrm{D4}$ & NM 0 & & & 18 \\
\hline & 1 & & & 7 & IAD4 & NM_0 & & 97 & 18 \\
\hline & I_C & & & & $\mathrm{AD} 4$ & 1 & & & 18 \\
\hline IET & NM_000245 & & 26 & 7 & MAD4 & NM_00 & & & 18 \\
\hline & $1 \_00$ & & & 7 & 1AD4 & NM_0 & 706 & 329 & 18 \\
\hline RAF & NM_00 & & & 7 & MAD4 & NM_0C & & 289 & 18 \\
\hline BRAF & NM_004333 & 140481372 & 140481496 & 7 & MAD4 & NM_00 & & 979 & 18 \\
\hline R1 & 0 & & & & & $\mathrm{~N}$ & & & 18 \\
\hline GFR1 & NM_015850 & & & - & MAD4 & NM_00 & & 48603149 & 18 \\
\hline FGFR1 & NM_015850 & 38279311 & 38279462 & 8 & SMAD4 & NM_005359 & 48604622 & 48604839 & 18 \\
\hline
\end{tabular}


Table 2. The list of RNA and DNA targets in a 62-gene lung cancer panel.

RNA targets

\begin{tabular}{|c|c|c|}
\hline Gene & Exon & $\begin{array}{c}\text { Sense } \\
\text { /antisense }\end{array}$ \\
\hline ALK & 18 & antisense \\
\hline ALK & 19 & antisense \\
\hline ALK & 20 & antisense \\
\hline ALK & 21 & antisense \\
\hline $\mathrm{B} 2 \mathrm{M}$ & 2 & antisense \\
\hline B2M & 2 & sense \\
\hline BRAF & 8 & antisense \\
\hline BRAF & 9 & antisense \\
\hline BRAF & 10 & antisense \\
\hline BRAF & 11 & antisense \\
\hline BRAF & 12 & antisense \\
\hline CD274 & 2 & antisense \\
\hline CD274 & 3 & antisense \\
\hline $\mathrm{CD} 274$ & 4 & antisense \\
\hline CD274 & 4 & sense \\
\hline $\mathrm{CD} 274$ & 5 & sense \\
\hline CD274 & 6 & antisense \\
\hline CHMP2A & 3 & antisense \\
\hline CHMP2A & 3 & sense \\
\hline CSF1R & 9 & antisense \\
\hline CSF1R & 10 & antisense \\
\hline CSF1R & 11 & antisense \\
\hline CSF1R & 12 & antisense \\
\hline CSF1R & 13 & antisense \\
\hline CSF1R & 14 & antisense \\
\hline CTLA4 & 3 & antisense \\
\hline EGFR & 1 & antisense \\
\hline EGFR & 8 & antisense \\
\hline EGFR & 9 & antisense \\
\hline EGFR & 23 & sense \\
\hline EGFR & 24 & sense \\
\hline EGFR & 25 & sense \\
\hline ESR1 & 3 & sense \\
\hline ESR1 & 4 & sense \\
\hline ESR1 & 5 & sense \\
\hline ESR1 & 6 & sense \\
\hline ETV6 & 1 & sense \\
\hline ETV6 & 2 & antisense \\
\hline ETV6 & 2 & sense \\
\hline ETV6 & 3 & antisense \\
\hline ETV6 & 3 & sense \\
\hline ETV6 & 4 & sense \\
\hline ETV6 & 5 & antisense \\
\hline ETV6 & 5 & sense \\
\hline ETV6 & 6 & antisense \\
\hline ETV6 & 6 & sense \\
\hline ETV6 & 7 & antisense \\
\hline FGFR1 & 2 & antisense \\
\hline FGFR1 & 3 & antisense \\
\hline FGFR1 & 4 & antisense \\
\hline FGFR1 & 5 & antisense \\
\hline FGFR1 & 6 & antisense \\
\hline FGFR1 & 7 & antisense \\
\hline FGFR1 & 8 & antisense \\
\hline FGFR1 & 9 & antisense \\
\hline FGFR1 & 10 & antisense \\
\hline FGFR1 & 11 & antisense \\
\hline FGFR1 & 17 & sense \\
\hline FGFR2 & 2 & antisense \\
\hline FGFR2 & 8 & antisense \\
\hline FGFR2 & 9 & antisense \\
\hline FGFR2 & 10 & antisense \\
\hline FGFR2 & 17 & sense \\
\hline FGFR3 & 8 & antisense \\
\hline FGFR3 & 9 & antisense \\
\hline FGFR3 & 10 & antisense \\
\hline FGFR3 & 17 & sense \\
\hline
\end{tabular}

DNA targets

\begin{tabular}{|c|c|c|c|c|c|}
\hline \multirow{2}{*}{$\begin{array}{l}\text { Gene } \\
\text { FGFR4 }\end{array}$} & \multirow{2}{*}{$\begin{array}{c}\text { Exon } \\
2\end{array}$} & \multirow{2}{*}{$\begin{array}{c}\text { Sense } \\
\text { /antisense } \\
\text { antisense }\end{array}$} & \multicolumn{3}{|c|}{ Selected hotspot exons } \\
\hline & & & ABL1 & FLT3 & MPL \\
\hline FGFR4 & 8 & antisense & AKT1 & FOXL2 & NRAS \\
\hline FGFR4 & 9 & antisense & $\mathrm{APC}$ & GNA11 & PIK3CA \\
\hline FGFR4 & 10 & antisense & ATM & GNAQ & POLE \\
\hline FGFR4 & 17 & sense & AURKA & GNAS & PTEN \\
\hline FGR & 1 & antisense & CDK4 & $\mathrm{H} 3 \mathrm{~F} 3 \mathrm{~A}$ & PTPN11 \\
\hline FGR & 2 & antisense & CDKN2A & HRAS & RHOA \\
\hline FGR & 3 & antisense & CTNNB1 & IDH1 & SMAD4 \\
\hline GZMA & 2 & antisense & ERBB2 & IDH2 & TERT \\
\hline GZMA & 3 & antisense & ERBB3 & JAK2 & TP53 \\
\hline GZMA & 4 & antisense & EZH2 & KIT & \\
\hline GZMA & 5 & antisense & FBXW7 & KRAS & \\
\hline
\end{tabular}

$\begin{array}{lll}\text { MET } & 13 & \text { antisense } \\ \text { MET } & 14 & \text { antisense }\end{array}$

MET $15 \quad$ antisense

NRG1 1 antisense

NRG1 2 antisense

NRG1 3 antisense

NRG1 4 antisense

NRG1 6 antisense

NTRK1 8 antisense

NTRK1 10 antisense

NTRK1 11 antisense

NTRK1 $12 \quad$ antisense

NTRK1 13 antisense

NTRK1 14 antisense

NTRK1 $15 \quad$ antisense

NTRK2 $11 \quad$ antisense

NTRK2 13 antisense

NTRK2 $14 \quad$ antisense

NTRK2 15 antisense

NTRK2 $16 \quad$ antisense

NTRK2 17 antisense

NTRK3 13 antisense

NTRK3 13 sense

NTRK3 $14 \quad$ antisense

NTRK3 $14 \quad$ sense

NTRK3 15 antisense

NTRK3 $15 \quad$ sense

NTRK3 $16 \quad$ antisense

PDCD1 2 antisense

PDCD1 3 antisense

PDGFRA 9 antisense

PDGFRA 10 antisense

PDGFRA 11 antisense

PDGFRA 12 antisense

PDGFRA 13 antisense

PDGFRA 14 antisense

PRF1 2 antisense

PRF1 3 antisense

RET 8 antisense

RET 9 antisense

RET 10 antisense

RET 11 antisense

RET 12 antisense

RET 13 antisense

ROS1 31 antisense

ROS1 32 antisense

ROS1 33 antisense

ROS1 34 antisense

ROS1 35 antisense

ROS1 36 antisense

ROS1 37 antisense

TRAC 1 antisense

TRBC 1 antisense


a. Anchored multiolex target enrichment
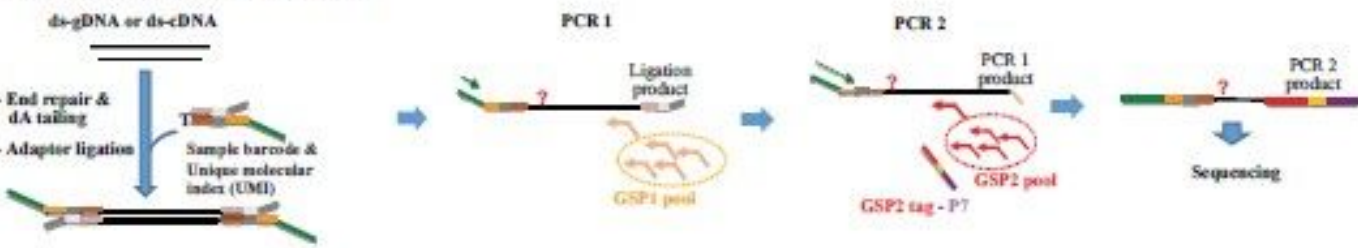

b. Reference aligument \& de-duplication

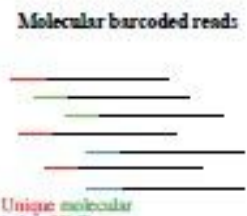

Unigue nolbo
inder fiTM?

Raw EASTO

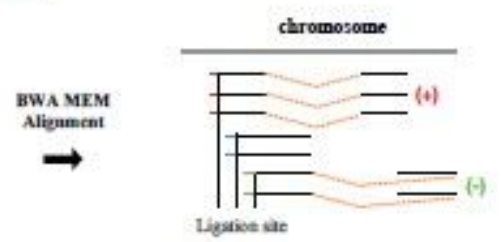

Ram BAM
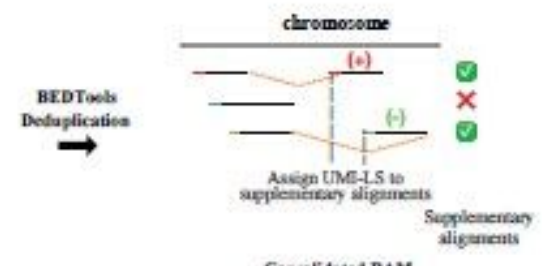

c. CIGAR transformation \& candidate breakpoint calling

Sepplesenentan alignatits
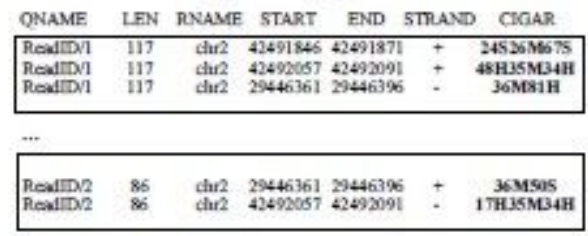

S. vel clip, M mappes, H. loxt clip

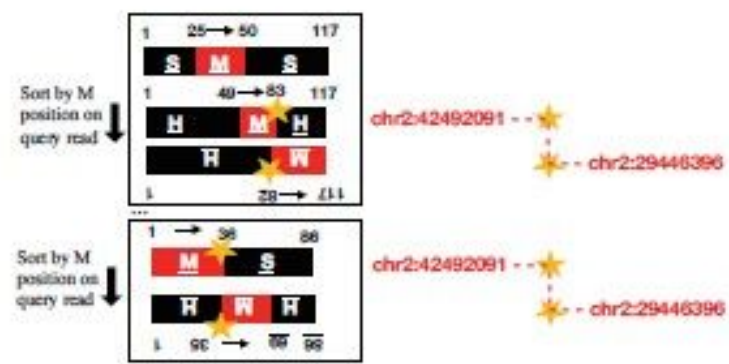

d. Initial brealpoint filtering

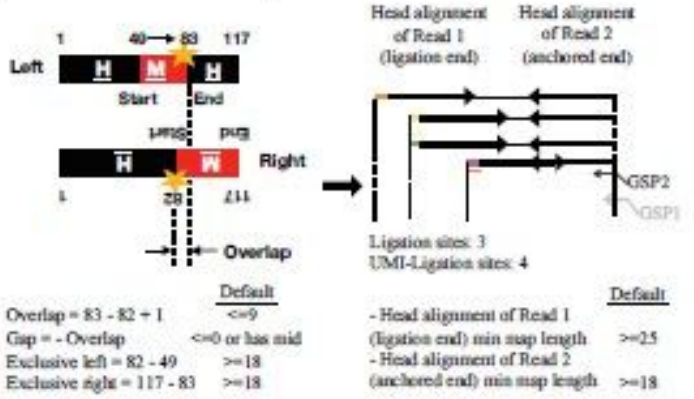

e. Brealspoint gene annotation

f. In-frame calculation

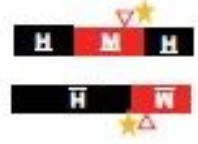

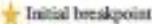

$\triangle$ Anovialed

Lef anemetatisa positio:

- bresiepoint-1Ovetsop +6)

Rigle sanososon pesition

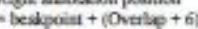

\section{5 cDNA positine A (pA)}
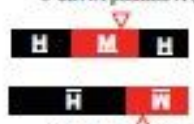

3' CDNA Posilion B (1)

CDA position fante divexe (D) - (pB $\$ 3$ - (pA - overbep $\% 3) \leqslant 3$ tr $\mathrm{D}=1$, thes in-finane

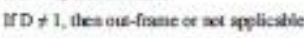

g. Eron-boundary alignment calcolation

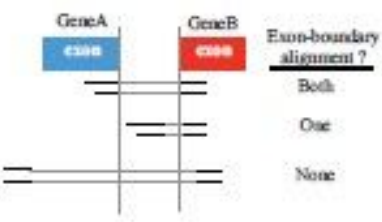

\section{h. Resnlt reporting \& visualization}

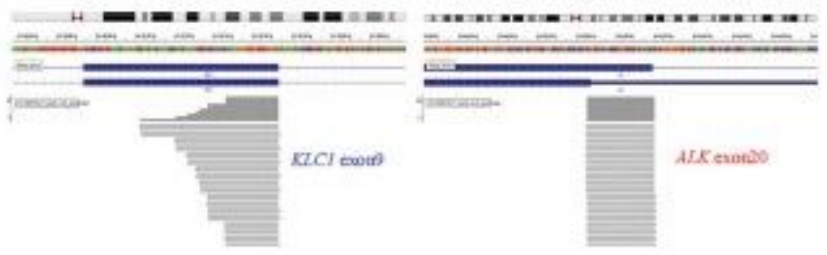

\section{Figure 1}

The framework of SplitFusion algorithm design. (a) Next-generation sequencing (NGS) library construction using the anchored multiplex PCR. Double-stranded CDNA and gDNA were subjected to endrepair, dA-tailing, and ligation of a half-functional $Y$ adaptor, which contains sample barcode and unique molecular identifier (UMI). Two rounds of hemi-nested multiplex PCR reactions were used to enrich any 
sequences (denoted in red question marks) downstream of the gene specific primers (GSPs), resulting in NGS library for sequencing. (b) NGS FASTQ data was de-multiplexed by sample barcodes, followed by adaptor trimming. Different colors represent different UMIs tagged to different molecules. The data was aligned to reference using BWA-MEM, and de-duplicated based on a combination of unique UMI and ligation site (UMI-LS). Supplementary alignments were assigned with the same UMI-LS as their Read 1 starting alignments. Reads with supplementary alignments (SA) were extracted. (c) The CIGARs of reads with supplementary alignments were transformed. For the same read ID, all alignments were sorted by their mapping positions (M) on the query read, and the candidate breakpoint(s) was inferred. (d) The initial candidate breakpoints were filtered by maximum overlapping length, maximum gap, minimum exclusive mapping lengths of partner alignments, and the minimum mapping lengths of ligation end and anchored end alignments; and further subjected to gene annotation (e), frame-ness calculation (f), exonboundary alignment judgement (g), and reporting and visualization (h).

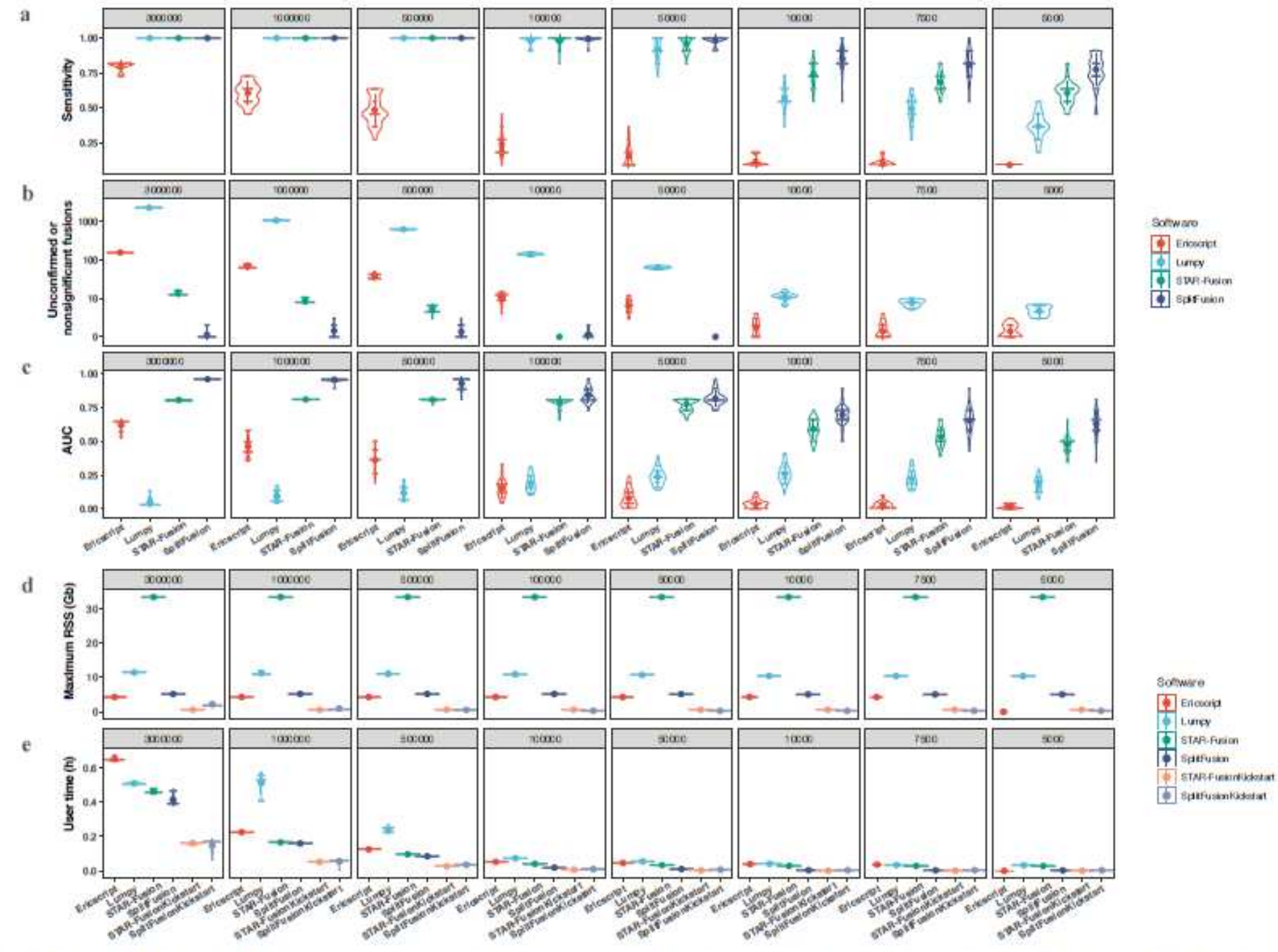

\section{Figure 2}

Benchmarking sensitivity, specificity, accuracy, computation memory use and speed among the four software - Ericscript, Lumpy, STAR-Fusion and SplitFusion. The datasets of the 11 positive samples were 
randomly down-sampled to different sizes (reads: $3 \mathrm{M}, 1 \mathrm{M}, 500 \mathrm{~K}, 100 \mathrm{~K}, 50 \mathrm{~K}, 10 \mathrm{~K}, 7.5 \mathrm{~K}$ and $5 \mathrm{~K}$ ), each size with 21 replicates (random seeds), generating 1,848 datasets for analyses by the four software with their default parameters using one computation thread. (a) Sensitivity was calculated as the fraction of samples resulted in expected gene fusions (ALK/EWSR1) among the 11 samples of given size and given random seed. (b) Specificity was evaluated by the number of unconfirmed or non-significant fusions, i.e. other than ALK and EWSR1. (c) The overall accuracy was calculated by the area under the precision-recall curve (AUC) at varied supporting read thresholds (from minimum to maximum supporting reads) according to the previous method. The violin outlined the distribution (the solid circle was the mean) of the statistics for each group (21 datasets). (d) Maximum RSS: the maximum resident set size (RSS: the portion of memory occupied by a process that is held in main memory) during the computation of the software. (e) User time: the amount of total CPU time for a given analysis.

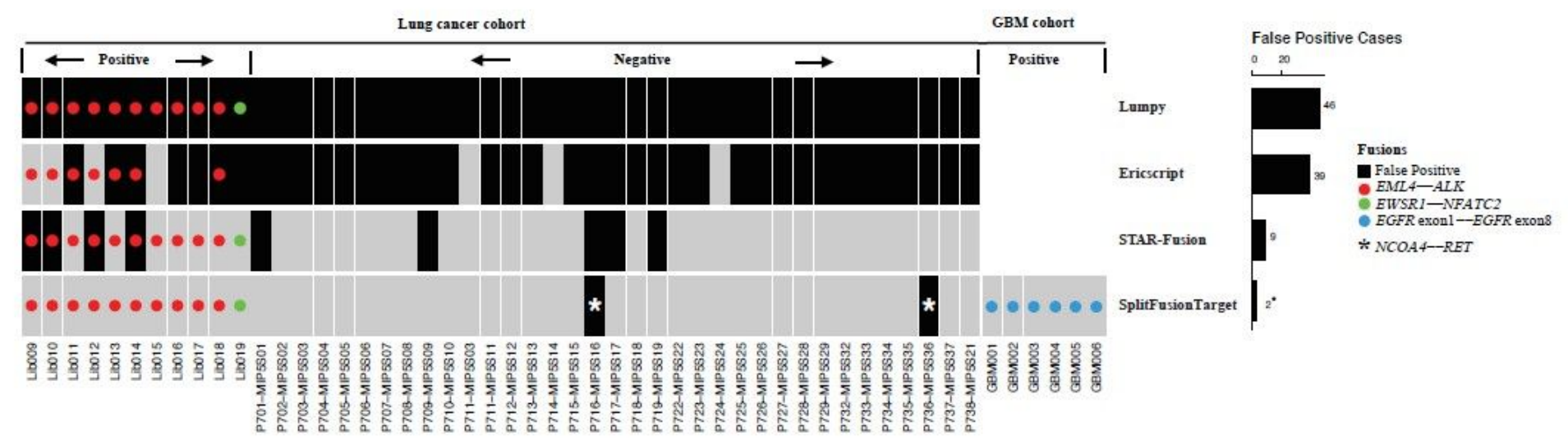

Figure 3

Fusion calling results for the 46 lung cancer samples with clinical known diagnoses ( 11 positive and 35 ALKnegative) by the four software - Ericscript, Lumpy, STAR-Fusion and SplitFusion. Each column represented one sample. Different colors and symbols denoted different fusions. Total number of falsepositive calls (other than ALK/EWSR1) for each software was plotted in the barplot. Out of 35 cases of glioblastoma (GBM), six were found with EGFR vIll using SplitFusion-Target. The GBM samples were not subjected for analysis by the other three software. 

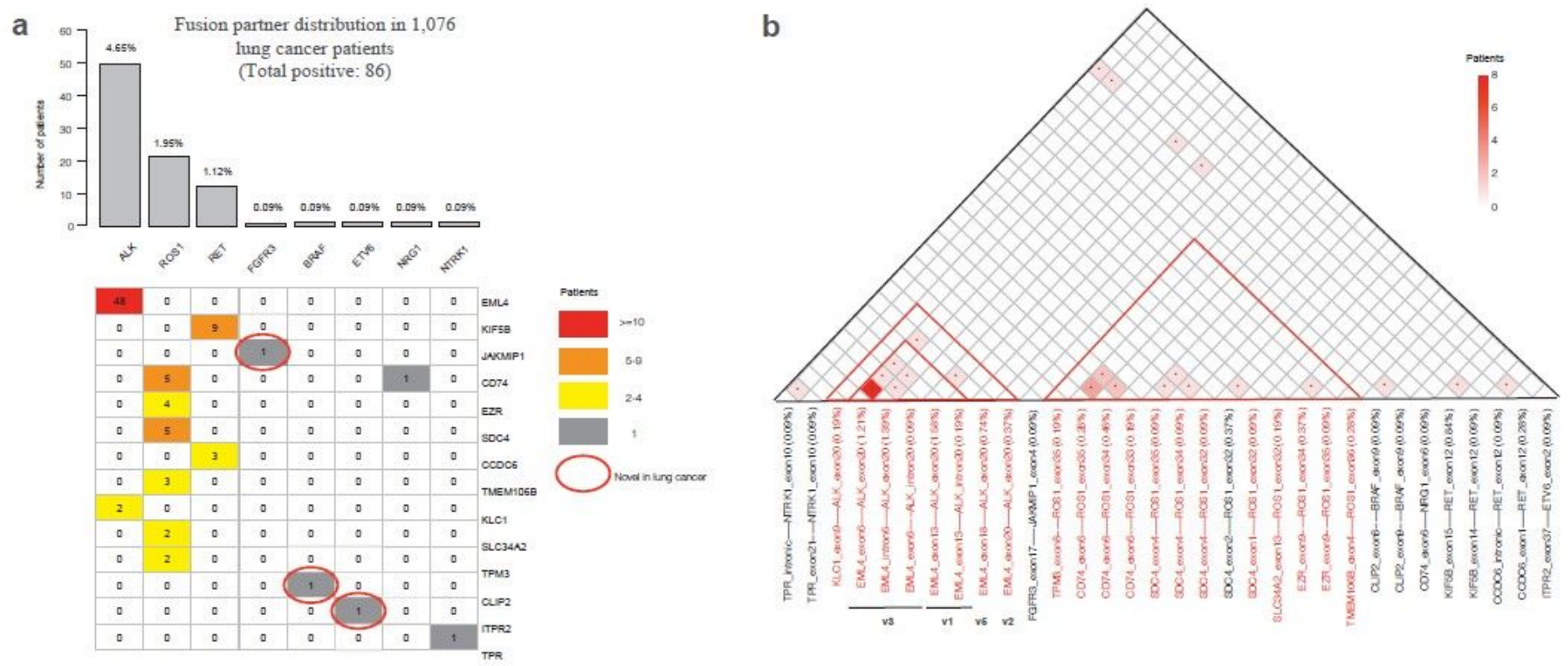

\section{Figure 4}

The analysis of 1,076 clinical lung cancer formalin-fixed paraffin-embedded samples using SplitFusion. (a) The prevalence of different target gene fusions were shown in the barplot. In total, 86 (8.0\%) samples were fusion-positive. The numbers of target genes with partner genes (right column) were indicated in the cells. (b) The numbers of co-occurrence of fusions in the same samples were plotted in the matrix. The EML4-ALK fusion variants were denoted under each specific fusion. 
a

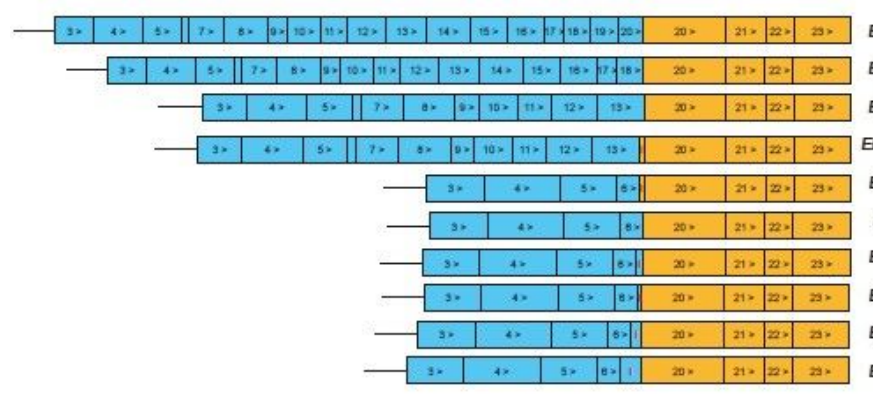

b

EML4 exon20 - ALK exon20 (V2) EML4 exon18 - ALK exon20 (v5) EML4 exon13 - ALK exon20 (V1) EML4 exon13 - ALK intron 20 (V1b) EML4 exon6 - ALK intron20 (V3a) EML4 exon6 - ALK exon20 (v3b) EML4 intron6 - ALK exon20 (v3c) EML4 intron6 - ALK exon20 (v3d) EML4 intron6 - ALK exon20 (v3e) EML4 intron6 - ALK exon20 (v3f)

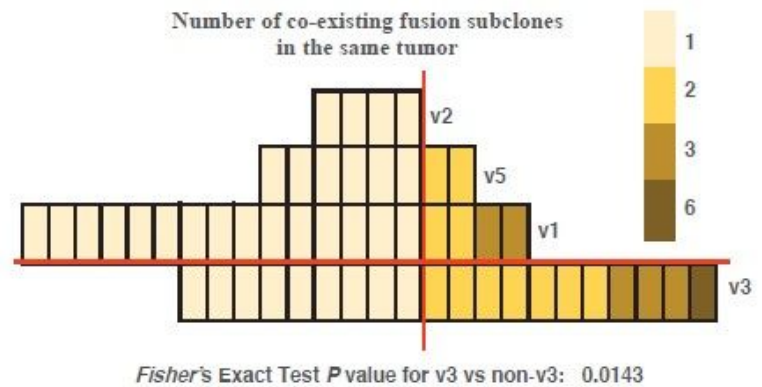

Fisher's Exact Test $\boldsymbol{P}$ value for v3 vs non-v3: 0.0143

c
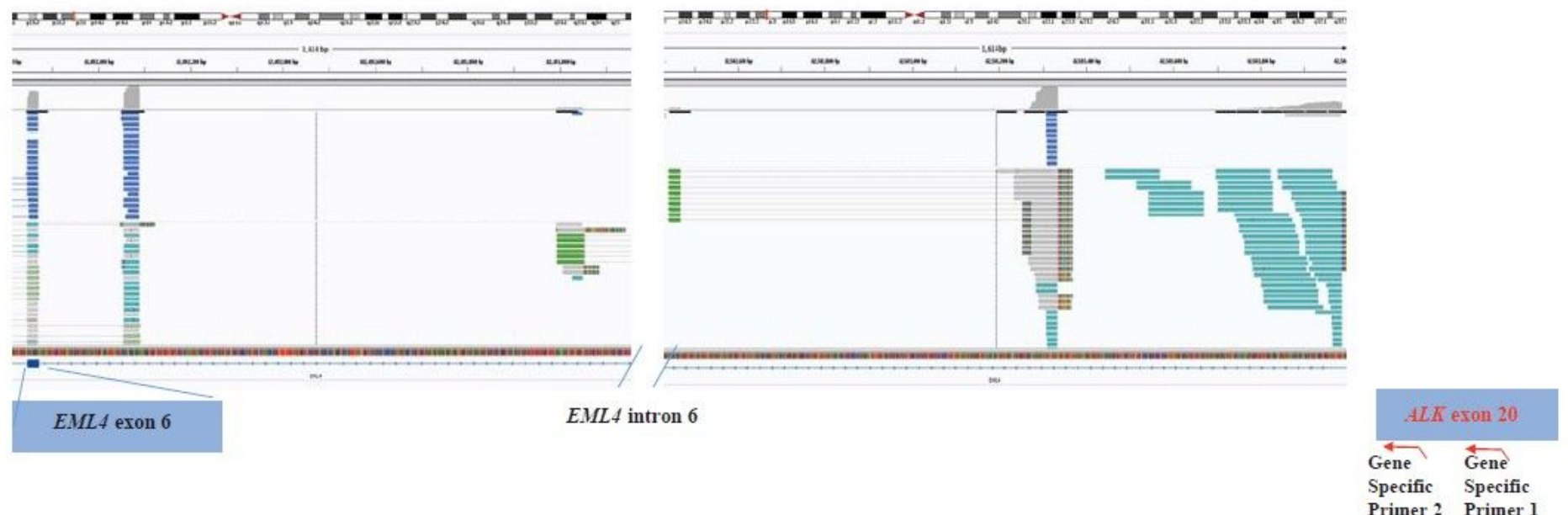

Figure 5

The analysis of breakpoint-defined subclones in EML4-ALK positive lung tumors. (a) The schematic representations of different EML4-ALK fusion variants. Blue cells represent EML4 exons and yellow cells represent ALK exons. Numbers inside the cells showed exon numbers. Cells with red strokes represents introns. (b) Each tile represents one patient. The tile color gradients denote the number of co-existing fusion subclones, defined by exact breakpoint, in the same tumor. (c) For one EML4-ALK variant 3 lung tumor sample, the Integrated Genome Viewer (IGV) showed 6 fusion breakpoints in EML4 exon 6 and intron 6 . Their gene partner ALK exon 20 was schematically shown on the right in red, and with nested gene specific primers 1 and 2 .

\section{Supplementary Files}

This is a list of supplementary files associated with this preprint. Click to download.

- FigureS1.jpg

- Figures2.jpg

- FigureS3.jpg

- FigureS4.jpg 
- FigureS5.jpg

- Table1.png

- Table2.png 